\title{
Permeability of fibrous carbon materials
}

3

4

5

6

7

8

9

10

11

12

13

14 Jean TOUTAIN :

15

16 Fouzia ACHCHAQ :

17

18 Philippe LEGROS :

19

20

21

22

23

24

25

Vanessa FIERRO

${ }^{1}$ Université de Lorraine, CNRS, IJL, F-88000 Epinal, France

${ }^{2}$ I2M - TREFLE department, ENSAM, 33405 Talence cedex, France

${ }^{3}$ PLACAMAT, 33608 Pessac cedex, France

Blagoj KARAKASHOV : $\quad$ Orcid: https://orcid.org/0000-0002-1320-9191

e-mail : blagoj.karakashov@univ-lorraine.fr

Orcid : https://orcid.org/0000-0002-9891-3582

e-mail : jean.toutain@enscbp.fr

Orcid : https://orcid.org/0000-0001-7966-5352

e-mail: fouzia.achchaq@u-bordeaux.fr

Orcid : https://orcid.org/0000-0003-2839-733X

e-mail : philippe.legros@u-bordeaux.fr

Orcid : https://orcid.org/0000-0001-7081-3697

e-mail : vanessa.fierro@univ-lorraine.fr

Alain CELZARD: $\quad$ Orcid: https://orcid.org/0000-0003-0073-9545

e-mail: alain.celzard@univ-lorraine.fr

\footnotetext{
* Corresponding author. Tel: + 33372749614
} 
Abstract

The air permeability of a number of commercial fibrous carbon materials: soft non-woven 28 felts, rigidized felts and rigid boards, based on either PAN- or Rayon-derived fibres 29 presenting various diameters, graphitised or not, and consolidated by different methods, was measured and investigated. Consistent behaviours were found within families of closely

31 related materials, but the diversity of porous structures prevented any model, including the very popular Tomadakis-Sotirchos equation, to fit all results. The Archie's coefficient and the tortuosity factor for viscous flow were thus calculated. Not only all data were perfectly 34 aligned on one single master curve, but the analysis was extended to many other fibrous materials and the same master curve was found to be relevant. The Archie's coefficient thus appears to be an intrinsic property, purely defined by the material geometry, as it does not depend on the 1D, 2D or 3D-type of flow. A fitting equation was proposed, encompassing all fibrous materials in very broad ranges of porosities and porous structures.

40 Keywords: Carbon fibres; carbon felts; permeability; Archie's exponent 


\section{Introduction}

Flow and transport of fluids through porous media are of great interest for a wide range of engineering applications, such as petroleum industry, geology, agriculture, acoustics, chemical and biomedical engineering, and so on. As far as porous carbon materials are concerned, phenomena encountered in filters, adsorbents, catalyst supports, fuel cells, membranes, carbon foams used as heat sinks or as hosts for phase-change materials, and in the process of carbon fibres-based composites manufacturing, depend critically on permeability. The intrinsic permeability of a porous medium measures its ability of letting a fluid pass through it under the influence of a pressure gradient. For practical applications such as those listed above, it is of high interest to predict the permeability of a given medium based on its porous structure.

Many research works have been devoted to this topic in the past decades, combining experimental characterisation, simulation and modelling of porous media, as reported in several monographs [1-4]. But as far as carbon materials are concerned, published studies are quite scarce, despite the significance of the topic, especially in the field of electrochemistry in general, and of gas diffusion layers for fuel cells in particular. Indeed, and as far as the authors know, only a few works focused on the permeability changes of carbon felts either as a function of porosity, modified by axial compression $[5,6]$, or as a function of thickness $[7,8]$. Other papers just reported values of permeability of composite materials made of carbon felts coated with a microporous layer [9-11], or such that various amounts of PTFE were used as binder for gluing the fibres with each other [12-15].

In the former papers, obvious observations were noticed such as an increase of permeability with the open porosity of the medium, and then these trends were successfully accounted for by application of either the Kozeny-Carman (KC) equation or the TomadakisSotirchos (TS) equation. These two equations, as well as the various forms they may take, 
depending on the context, have been discussed in detail elsewhere [16]. The TS equation was found to be particularly suitable for describing the permeability of random fibre beds, not only because it describes numerical results of gas diffusion layers for fuel cells quite well [17], but also because it is supposedly is free of adjustable parameters [5].

However, all these studies lack a critical discussion of the corresponding parameters, whose values were predicted for a few ideal cases, but which cannot be used for all situations. This is especially the case of non-woven fibrous carbon materials, whose structures and physical properties are determined by the nature, the orientation distribution and the average diameter of the fibres, the type of bonding and the consolidation method, as well as the fabric architecture. Thus, these materials can present various needle-punching densities or, in most cases, be made of multi-layered randomly oriented fibre webs that were either mechanically or chemically consolidated. As a result, very different permeabilities can be measured at similar porosities, and vice-versa. In the present work, the static air permeability of no less than 18 different fibrous carbon materials was measured and fitted with the TS equation, with the aim of finding some potentially universal behaviour for the tortuosity. The paper is organised as follows. First, the fibrous carbons are described in terms of porosity, nature of the fibres, and structure of the mats. The way the through-plane permeability was measured is also detailed. Then, some reminders about the origin of the relevant permeability equations are given. The latter equations are applied to the studied materials and the obtained data are presented and discussed. Finally, a universal curve linking the bulk tortuosity to the so-called Archie's exponent is presented, which should encompass any other non-woven fibrous material, carbonaceous or not. 


\section{Materials and methods}

93

\subsection{Materials}

It is useful, in the context of the present work, to recall how fibrous carbon mats are obtained, and how the various ways of preparing them may impact their final structural and physical properties such as porosity and bulk density, mechanical, electrical and thermal properties, and, of course, permeability and fluid flow properties in general. As it is usually the case [18], two types of precursors were used for producing the present carbon nonwovens: polyacrylonitrile (PAN) and regenerated cellulose (Rayon). From the latter fibrous precursors, non-wovens can be designed by various methods leading to different bond structures, whose type, shape, rigidity, size and density influence the materials properties. The choice of the aforementioned parameters mainly depends on the foreseen application.

One way of manufacturing mechanically bonded non-wovens is interlacing either individual fibres or loose fibrous strands. These kinds of connections are flexible, and the constituent fibres are able to slide or move within the bonding joints. Typically, periodic needle-punched fabrics have their unique structural architecture caused by the interaction of fibres with the needle barbs of the needle-punching machine, see Fig. 1. By reorientation and migration of fibre segments from the surface of the web towards the interior of the fabric, new pillars of fibre segments are rearranged roughly perpendicular to the bedding plane of the fabric fibres. Carbon non-wovens layers are thus reinforced by the formation of such rearranged fibrous pillars, and hence the layers are compacted and interlocked with each other $[19,20]$. Production parameters that influence the bonding points are: dimensions/number of barbs on the needles in relation to the fibre diameter, needle-punching density, and needle penetration. 

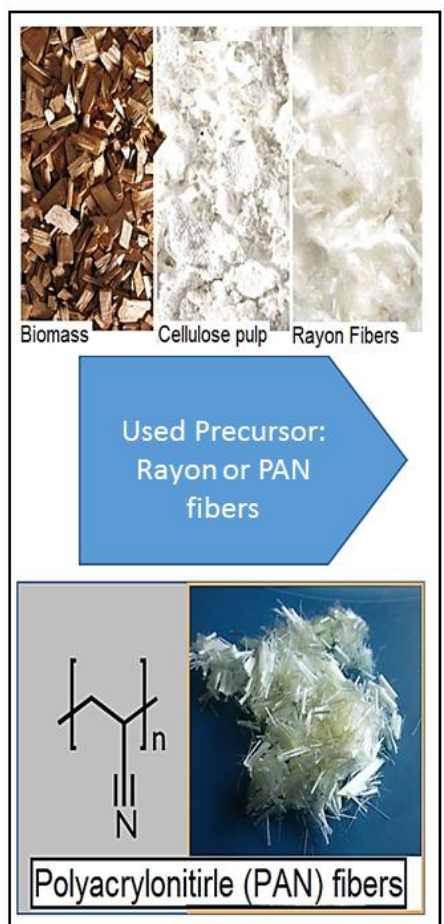
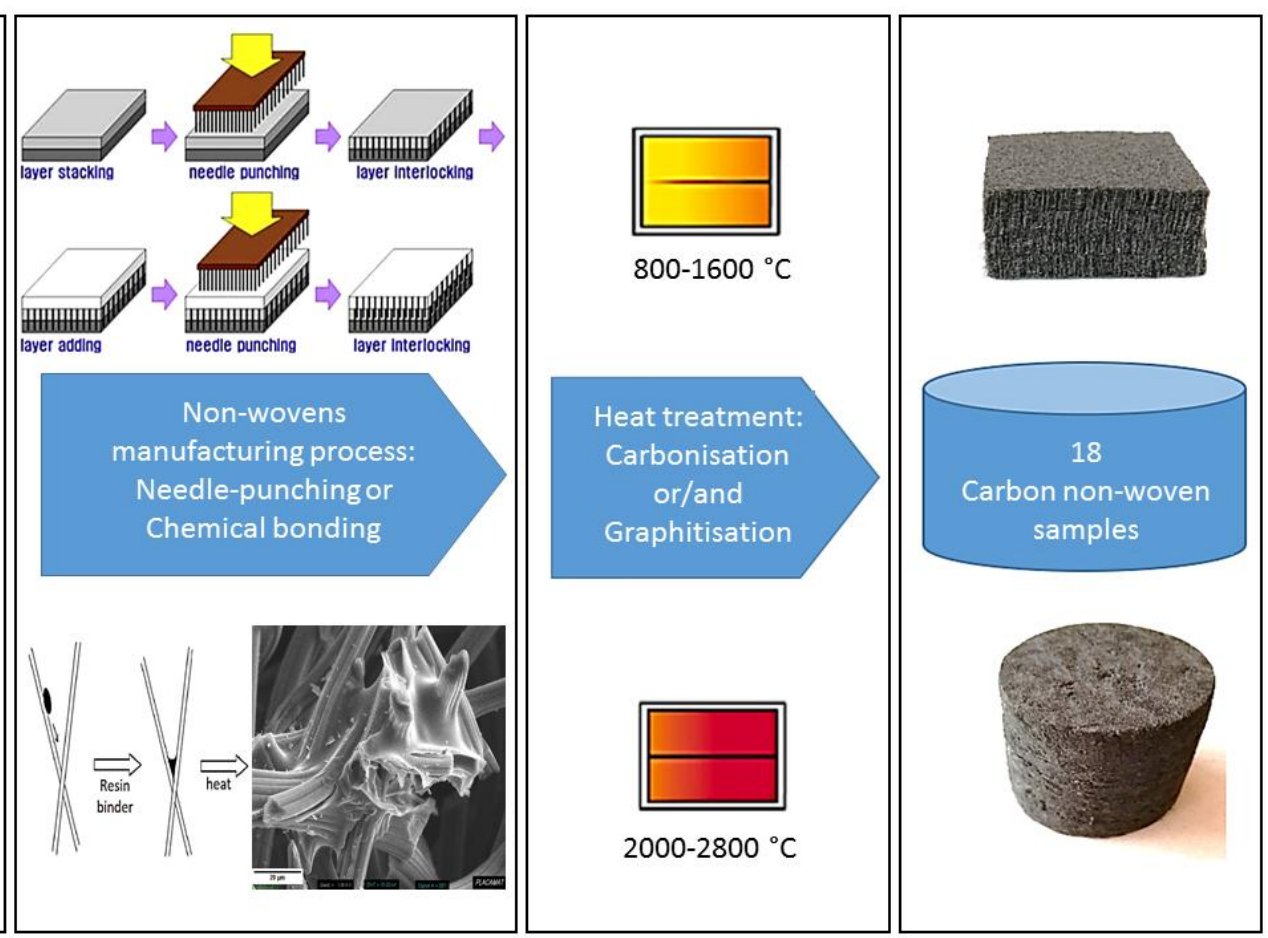

Fig. 1. Overview of carbon non-wovens production.

117 Some of the resultant needle-punched non-wovens can be then infiltrated with solutions of

118 phenolic resin at various concentrations [21], thereby giving them an additional chemical

119 consolidation. Rigidized carbon felts are thus obtained, which have a modified morphology with respect to the initial soft materials, and hence exhibit modified physical properties.

In contrast, chemically-bonded fabrics are formed by adhesion or cohesion between

122 polymer surfaces. A minor part of the fibrous network thus becomes rigid as the freedom of

123 movements within the bond points and the fibres is significantly reduced (see again Fig. 1)

124 [22-24]. The delivery of the resin in the fabric is largely ruled by the way it is introduced into

125 the web and how it flows in-between the fibres, but $5 \mathrm{wt} \%$ of binder is often enough to bond

126 them at their surfaces and interconnections [25]. The resultant physical properties thus depend

127 on fibres, resin and interaction between them, and on their relative spatial arrangement,

128 surface and bulk properties [26]. Since a large number of fibres may be entangled and joined

129 by a coating of binder connecting fibre crossover points and/or interfibrous porosity, this 
130 results in solid bond points in most of fabrics, making these materials quite different from

131 their needle-punched counterparts.

132 After the non-woven formation process, the materials are thermally treated at temperatures

133 typically in the ranges of $800-1600^{\circ} \mathrm{C}$ for carbonisation, or of $2000-2800^{\circ} \mathrm{C}$ for graphitisation

134 [27,28]. Although carbonised and graphitised non-wovens exhibit exactly the same 135 appearance, the use of different precursors and manufacturing parameters changes the final 136 non-wovens properties. There are indeed several commercial grades of carbon/graphite non137 wovens available from different manufacturers, with different end-uses, and the differences 138 from one material to another can be considerable.

139 Thus, in the present work, 18 commercial samples were received from 5 different 140 suppliers, most of them having properties unknown to us. They were first sorted into various 141 groups and sub-groups for an easier presentation and discussion of the characterisation data, 142 see Table 1. The samples were labelled as either soft felt, rigidized soft felt or rigid board (SF, $143 \mathrm{rSF}$ and $\mathrm{RB}$, respectively), followed by a letter related to the final heat-treatment temperature

144 ( $\mathrm{C}$ for carbonisation or $\mathrm{G}$ for graphitisation), plus a number referring to the precursor (1 for 145 Rayon or 2 for PAN) and a small letter corresponding to the fibre diameter (a for thin fibres or $146 \mathrm{~b}$ for thick fibres). The last, one or two, capital letters consider the samples manufacturing 147 origin. For instance, $\mathrm{SFC} 1 \mathrm{aC}$ refers to a soft felt, carbonised at $1200^{\circ} \mathrm{C}$, originating from a 148 Rayon preform made of thin fibres (diameter 9-12 $\mu \mathrm{m}$ ), kindly provided from one of the 149 commercial suppliers (assigned as "C" for "CeraMaterials"). Table 1 gives also reference to 150 the commercial supplier codes of the investigated materials. 


\begin{tabular}{|c|c|c|c|c|}
\hline $\begin{array}{l}\text { Type of carbon fibre } \\
\text { material }\end{array}$ & Precursor & Fibre diameter & Sample code used here & Commercial name and supplier \\
\hline \multirow{14}{*}{$\begin{array}{l}\text { Needle-punched } \\
\text { non-woven } \\
\text { Soft Felts }\end{array}$} & \multirow{7}{*}{ Rayon } & \multirow{7}{*}{$9-12 \mu \mathrm{m}$} & SFC1aC & Carbon (Rayon) felt CeraMaterials \\
\hline & & & SFG1aC & Graphite (Rayon) felt CeraMaterials \\
\hline & & & SFC1aBG & RSF1 Beijing Great Wall Co. \\
\hline & & & SFG1aBG & RSF2 Beijing Great Wall Co. \\
\hline & & & SFG1aSC & GF2 Schunk \\
\hline & & & SFC1aSI & SIGRATHERM ${ }^{\circledR}$ KFA5 \\
\hline & & & SFG1aSI & SIGRATHERM $^{\circledR}$ GFA10 \\
\hline & \multirow{7}{*}{ PAN } & \multirow{3}{*}{$9-10 \mu \mathrm{m}$} & SFG2aZF & PX 35 ZOLTEK $^{\mathrm{TM}}$ \\
\hline & & & SFG2aC & GFE-1 CeraMaterials \\
\hline & & & SFG2aBG & $\begin{array}{c}\text { Battery Energy Saving Felt Beijing } \\
\text { Great Wall Co. } \\
\end{array}$ \\
\hline & & \multirow{4}{*}{$17-20 \mu \mathrm{m}$} & SFC2bC & Carbon (PAN) felt CeraMaterials \\
\hline & & & SFG2bC & Graphite (PAN) felt CeraMaterials \\
\hline & & & SFC2bBG & PSF1 Beijing Great Wall Co. \\
\hline & & & SFG2bBG & PSF2 Beijing Great Wall Co. \\
\hline \multirow{2}{*}{$\begin{array}{l}\text { Chemically rigidized needle- } \\
\text { punched } \\
\text { non-woven Soft Felts } \\
\end{array}$} & \multirow[b]{2}{*}{ PAN } & \multirow[b]{2}{*}{$17-20 \mu \mathrm{m}$} & rSFG2bBG & PRF3 Beijing Great Wall Co. \\
\hline & & & rSFC2bC & $\begin{array}{c}\text { Carbon Insulating Board } \\
\text { CeraMaterials } \\
\end{array}$ \\
\hline \multirow{2}{*}{$\begin{array}{l}\text { Chemically bonded } \\
\text { rigid boards }\end{array}$} & \multirow{2}{*}{ Rayon } & \multirow{2}{*}{$9-12 \mu \mathrm{m}$} & RBG1aSI & SIGRATHERM $^{\circledast}$ MFA \\
\hline & & & RBG1aC & $\begin{array}{l}\text { Graphite Insulating Board } \\
\text { CeraMaterials } \\
\end{array}$ \\
\hline
\end{tabular}

Table 1. Sorting of 18 commercial non-woven carbon materials investigated here into groups and sub-groups, depending on their main characteristics. 


\subsection{Methods}

\subsubsection{Porosity and morphology of samples}

The porosity $\varepsilon$ (dimensionless) of all carbon non-woven samples was calculated from Eq. (1), where $\rho_{b}\left(\mathrm{~cm}^{3} \mathrm{~g}^{-1}\right)$ and $\rho_{s}\left(\mathrm{~cm}^{3} \mathrm{~g}^{-1}\right)$ are bulk and skeletal densities, respectively:

$$
\varepsilon=1-\frac{\rho_{b}}{\rho_{s}}
$$

$\rho_{s}$ was measured by helium pycnometry, using an AccuPyc II 1340 (Micromeritics, USA) automatic apparatus, whereas $\rho_{b}$ was determined by weighing 10 cylindrical samples and measuring their dimensions (thickness and diameter of felts or boards), then averaging the obtained results. The mass of the samples was measured with an analytical balance with an accuracy of $0.001 \mathrm{~g}$, and the diameter was determined with an electronic calliper with an accuracy of $0.01 \mathrm{~mm}$. The non-wovens thickness measurement was more problematic since soft felts are highly compressible, and using a calliper can lead to erroneous results [29]. The thickness of nonwoven materials, defined as the distance between the upper and lower surfaces of the material measured under a specified pressure [30], is normally measured as the gap between a flat solid surface and a presser foot or other measuring instrument used to apply a known, moderate pressure.

Herein, the thickness of soft carbon felts was measured by using an Instron 5944 universal testing machine equipped with a $2 \mathrm{kN}$ load cell, and applying a modified ASTM D572997(2004)e $1^{1}$ standard. The samples were placed between two parallel solid surfaces: a fixed lower one, and a vertically adjustable one. The thickness of each soft carbon felt was defined at a pressure described by the aforementioned standard (689 Pa for needle-punched materials) and then averaged by measuring 10 different samples of each material.

${ }^{1}$ ASTM D5729-97(2004)e1: Standard Test Method for Thickness of Nonwoven Fabrics | Engineering360 
The average fibre diameter and the overall fibrous materials morphology were investigated by Scanning Electron Microscopy (SEM), using a ZEISS EVO 50 instrument, equipped with an Everhart-Thornley secondary electron detector and operating at a maximum voltage of $30 \mathrm{kV}$. Carbon fibres can have circular or non-circular cross-sections [31], especially when derived from Rayon [32]. Therefore, the mean fibre diameter was obtained by drawing more than 10 segments across the cross-section, measuring their lengths and calculating the average value. The same procedure was repeated across at least 10 different fibres from 5 different locations in each sample to ensure a representative range of values. The corresponding measurements were carried out by using the ImageJ software. Even though this technique is restricted to the outer parts of the samples, it proved to be representative of the entire bulk since these (commercial) materials are usually reproducible and homogeneous [33].

\subsubsection{Average air permeability measurements}

The fluid flow through porous media such as non-woven fabrics follows Darcy's law, described by equation (2) [34]:

$$
\sigma=\frac{\Delta P A}{Q L}=\frac{R_{f}}{L}
$$

where the resistivity to fluid flow $\sigma\left(\mathrm{Pa} \cdot \mathrm{s} \mathrm{m}^{-2}\right)$ is calculated from the pressure drop $\Delta P(\mathrm{~Pa})$ and the flow rate $Q\left(\mathrm{~m}^{3} \mathrm{~s}^{-1}\right)$ of a fluid flowing across a material of section $A\left(\mathrm{~m}^{2}\right)$ and thickness $L(\mathrm{~m}) . R_{f}$ $\left(\mathrm{Pa} \cdot \mathrm{s} \mathrm{m}^{-1}\right)$ is called fluid flow resistance. Darcy’s law is broadly used in several engineering areas where the involved fluid is Newtonian with a low Reynolds number $(\operatorname{Re}<10)$. The inertial effects are thus not critical, so that the viscous interaction between the fluid and the porous solid is the main source of pressure drop [35]. 
The airflow resistance $R_{f}$, being the product $\sigma \times L$ according to Eq. (2), was directly measured with a Sigma airflow resistance meter (Mecanum, Canada). This apparatus (Fig. 2) was specially designed to measure the airflow resistance of materials within the range of $10-10^{6} \mathrm{~Pa} \cdot \mathrm{s} \mathrm{m}^{-1}$, and complies with two international standards: ISO 9053:1991 ${ }^{2}$ and ASTM C522-03(2016) ${ }^{3}$. The pressure regulator maintained the steady-state flow of air throughout the specimen, and a differential pressure transducer was used to quantify the static pressure difference between the free faces of the specimen with respect to atmosphere. Valid measurements were only made in the region of laminar airflow where, apart from random measurement errors, the airflow resistance (thus the airflow permeability) was strictly constant. Then, the airflow permeability $k\left(\mathrm{~m}^{2}\right)$ of all samples was calculated with the software SIGMA-X of the device, by application of Eq. (3):

$$
k=\frac{v L}{R_{f}}
$$

where $v=1.84 \times 10^{-5} \mathrm{~Pa}$ s is the dynamic viscosity of air at room temperature.

Because of their low thickness with respect to the required sample diameter, the fibrous mats were only characterised along their out-of-plane direction, i.e., perpendicular to the bedding plane of the fibres. For that purpose, cylindrical specimens having a diameter of $44.44 \mathrm{~mm}$ and a height imposed by the initial thickness of the materials were cut with a special round cutter so that the samples fitted tightly in the specimen holder, see Fig. 2. The measurements were carried out at 5 different airflow velocities, all below $50 \mathrm{~mm} \mathrm{~s}^{-1}$ (as recommended by the supplier as the upper limit for staying in non-turbulent regime), and the average value was calculated for each sample. Then, a series of 5 samples per type of fibrous carbon mat was investigated, and the average values and standard deviations were calculated.

2 ISO 9053:1991 : Acoustics - Materials for Acoustical Applications -- Determination of Airflow Resistance

${ }^{3}$ ASTM C522-03(2016) : Standard Test Method for Airflow Resistance of Acoustical Materials | Engineering360 
(a)

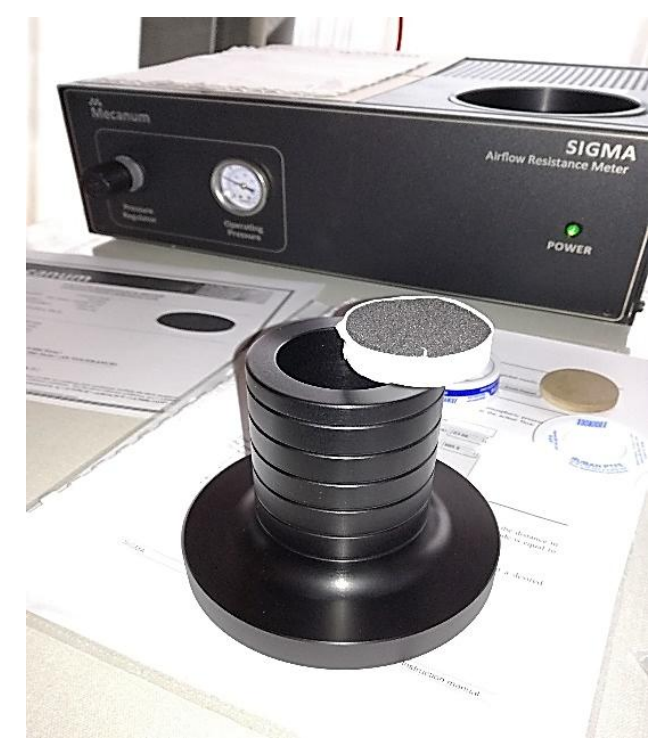

(b)

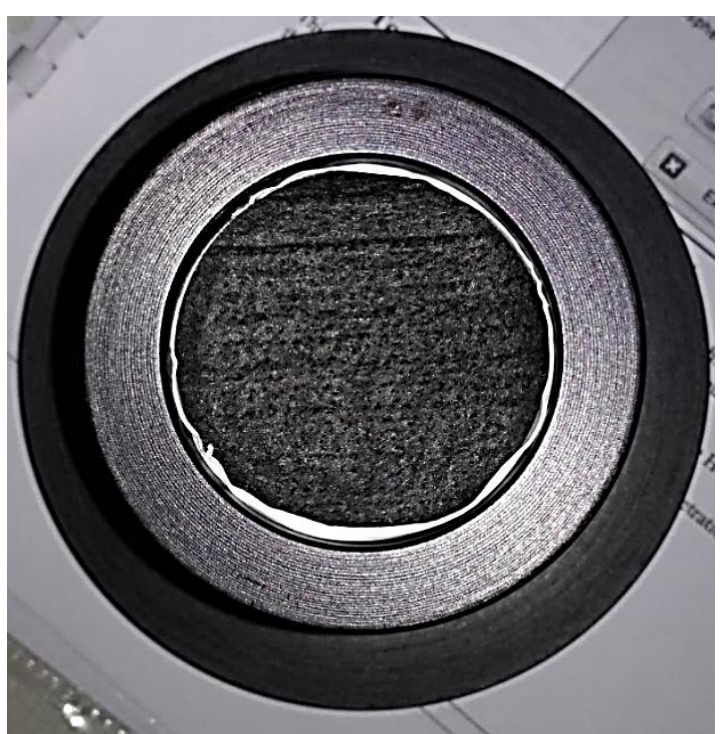

Fig. 2. (a) Prepared sample and sample holder for airflow resistance measurement; (b) top view of the sample holder, with one sample installed into it.

\section{Results}

\subsection{Materials structure and morphology}

One of the most remarkable characteristics of needle-punched nonwovens is their high porosity and large pore size [36]; these features were confirmed by SEM observations (see below and the Electronic Supplementary Information). Since various carbon felts were received from different suppliers, various ranges of bulk and skeletal densities, and of resultant total porosity, were expected and were indeed observed, see Table 2. The carbon non-wovens derived from PAN and Rayon precursors, regardless of fibre diameter, rigidity, or felt formation technique, exhibited porosity values varying from 87.5 to $95.7 \%$.

Needle-punched non-woven soft felts had clearly lower bulk density and higher porosity than rigidized soft felts and rigid boards, as a result of the chemical bonding process by which the latter 
materials were formed. The resin binder indeed accumulated at the intersection where fibres touch or nearly touch each other, thus bridging the gap between their surfaces. Consequently, the materials density slightly increased, thus making the total porosity decrease by $5 \%$ on average.

Table 2. Average fibre diameter (with standard deviation), total porosity, and air permeability in the out-of-plane direction (with standard deviation) of all fibrous carbons investigated here.

\begin{tabular}{|c|c|c|c|c|c|}
\hline \multirow{2}{*}{ Sample name } & \multicolumn{2}{|c|}{ Fibre diameter } & \multirow{2}{*}{$\begin{array}{l}\text { Total porosity } \\
\qquad(\%)\end{array}$} & \multicolumn{2}{|c|}{ Out-of-plane air permeability } \\
\hline & $(\mu \mathrm{m})$ & Std. dev. $(\mu \mathrm{m})$ & & $\left(\mathrm{m}^{2}\right)$ & Std. dev. $\left(\mathrm{m}^{2}\right)$ \\
\hline \multicolumn{6}{|c|}{ Rayon Needle-punched Soft Felts } \\
\hline SFG1aSC & 10.30 & 2.54 & 92.7 & $1.35 \times 10^{-10}$ & $3.68 \times 10^{-12}$ \\
\hline SFC1aBG & 11.98 & 1.59 & 93.9 & $8.41 \times 10^{-11}$ & $6.58 \times 10^{-13}$ \\
\hline SFG1aBG & 12.57 & 3.95 & 94.6 & $7.12 \times 10^{-11}$ & $3.49 \times 10^{-12}$ \\
\hline $\mathrm{SFC} 1 \mathrm{aC}$ & 12.02 & 2.34 & 94.8 & $8.07 \times 10^{-11}$ & $7.02 \times 10^{-13}$ \\
\hline SFG1aSI & 9.70 & 1.66 & 94.8 & $1.04 \times 10^{-10}$ & $7.51 \times 10^{-13}$ \\
\hline SFG1aC & 10.70 & 2.36 & 95.7 & $1.22 \times 10^{-10}$ & $3.34 \times 10^{-12}$ \\
\hline SFC1aSI & 9.59 & 3.00 & 95.7 & $1.26 \times 10^{-10}$ & $3.34 \times 10^{-12}$ \\
\hline \multicolumn{6}{|c|}{ PAN (thin fiber) Needle-punched Soft Felts } \\
\hline $\mathrm{SFC} 2 \mathrm{bBG}$ & 18.53 & 2.05 & 93.2 & $3.11 \times 10^{-10}$ & $1.31 \times 10^{-11}$ \\
\hline SFG2bBG & 17.98 & 1.83 & 93.3 & $2.90 \times 10^{-10}$ & $1.01 \times 10^{-11}$ \\
\hline SFG2bC & 19.71 & 2.15 & 93.8 & $3.33 \times 10^{-10}$ & $2.23 \times 10^{-12}$ \\
\hline $\mathrm{SFC} 2 \mathrm{bC}$ & 18.69 & 2.45 & 92.5 & $3.26 \times 10^{-10}$ & $7.38 \times 10^{-12}$ \\
\hline \multicolumn{6}{|c|}{ PAN (thick fiber) Needle-punched Soft Felts } \\
\hline SFG2aZF & 9.05 & 1.24 & 93.4 & $1.33 \times 10^{-10}$ & $6.66 \times 10^{-12}$ \\
\hline $\mathrm{SFG} 2 \mathrm{aC}$ & 9.06 & 1.49 & 95 & $1.77 \times 10^{-10}$ & $2.81 \times 10^{-12}$ \\
\hline SFG2aBG & 10.56 & 1.07 & 95.3 & $1.99 \times 10^{-10}$ & $5.62 \times 10^{-12}$ \\
\hline \multicolumn{6}{|c|}{ PAN (thick fiber) Rigidized Needle-punched Soft Felts } \\
\hline rSFG2bBG & 16.96 & 2.92 & 89.3 & $1.53 \times 10^{-10}$ & $7.09 \times 10^{-13}$ \\
\hline $\mathrm{rSFC} 2 \mathrm{bC}$ & 19.59 & 4.13 & 88.1 & $1.86 \times 10^{-10}$ & $2.13 \times 10^{-12}$ \\
\hline \multicolumn{6}{|c|}{ Rayon Rigid Boards } \\
\hline RBG1aC & 11.63 & 3.5 & 87.5 & $8.24 \times 10^{-11}$ & $6.86 \times 10^{-13}$ \\
\hline RBG1aSI & 10.01 & 1.34 & 89.6 & $5.47 \times 10^{-11}$ & $1.90 \times 10^{-12}$ \\
\hline
\end{tabular}


Evidence from SEM observations shows that all carbon non-wovens were anisotropic and made of randomly laid/dispersed fibre layers, consolidated by needle-punching and/or chemical bonding. All samples were quite correctly described by the schematic representations of either soft felt or rigid board shown in Fig. 3, in which $x$-, $y$ - and $z$ - axes are indicated. These directions originated from those of the machine with which the materials were prepared. The $x$ - and $y$ - axes are full equivalents and define the "in-plane" (IP) direction, i.e., the bedding plane of the fibres within which no preferential orientation exists. The orthogonal direction, $z$, is the "out-of-plane" (OP) direction of the materials.

(a)

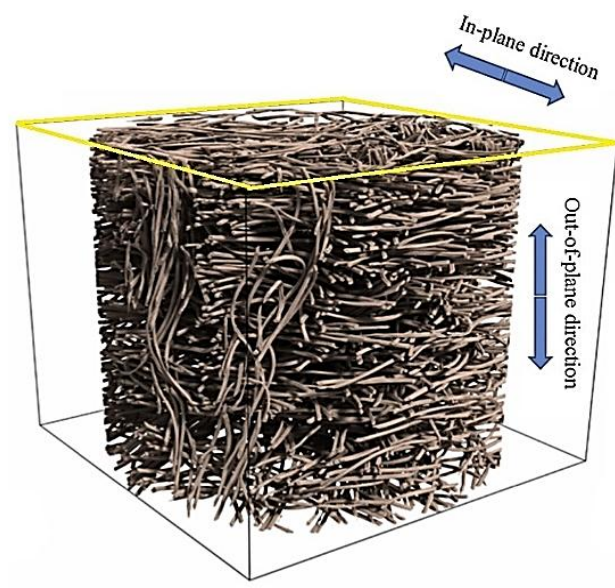

(b)

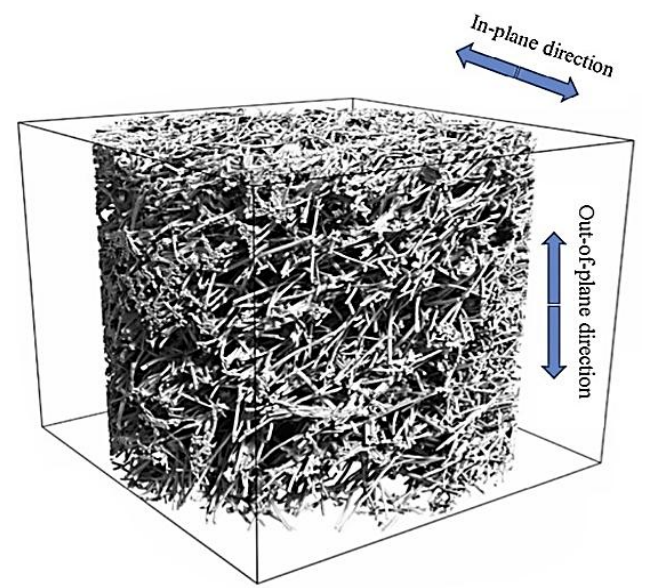

Fig. 3. Schematic representation of: (a) soft felt, and (b) rigid board samples, with $x-, y_{-}, z_{-}^{-}$ directions (reprinted from [37] with permission from Elsevier).

All soft carbon felts appeared to be organised according to a rather repetitive architecture, in which the IP-oriented fibres followed a defined pseudo-weaving pattern as a result of the fibres laying manufacturing step, prior to the needle-punching process. Part of the fibres directly exposed to the impact of needles and their barbs were abruptly redirected along the OP-direction. Other fibres were indirectly affected by the needle-punched redirected ones, and occasionally 
deviated from their initial orientation. Needling marks resulting from punching movements were also observed in the $x-y$ plane. The various commercial origins of the felts and the differences in fibre types and in assembly process resulted in soft felts with different morphologies, as seen in Figs. S1, S2 and S3 of the Electronic Supplementary Information.

The group of thick-fibre, PAN-derived rigidized felts had the same morphology as that of the aforementioned soft felts, except at the surface in the IP direction, see Fig. S4. The corresponding two samples were indeed additionally chemically bonded, thus discrete regions of rigidized fibre layers were formed. Such modification has two main motivations: (i) it is industrially used as upscaling technique for obtaining thicker materials by joining two or more needle-punched soft felt on top of each other (along the OP direction); (ii) it improves the mechanical properties by decreasing the probability of fibres release and movement.

On the other hand, the group of rigid boards presented completely different morphological characteristics: fibre intersections were bonded by more or less discrete regions made of glasslike carbon derived from phenolic resin. The materials investigated here had measured densities and porosities in the same ranges as observed elsewhere [24], reported to be $0.1-0.5 \mathrm{~g} \mathrm{~cm}^{-3}$ and $70-$ $90 \%$, respectively, for this kind of materials. They consisted of many clusters and bundles of fibres, due to the different manufacturing and chemical bonding processes, see Fig. S5 of the Electronic Supplementary Information. No clear needling pattern could be seen, unlike what was observed in all other groups of soft and rigidized soft felts.

As a final remark about the present fibrous carbon materials, it can also be recalled that the carbon fibre microstructure depends on precursor's properties and processing conditions, and different models were proposed to describe the cross-section fibre microstructures $[28,38]$. The average fibre diameters of all investigated non-woven carbon materials were analysed from the 
SEM pictures (two examples are shown in Fig. S6), as explained in subsection 2.2.1, and the corresponding values are given in Table 2. The materials could then be sorted in 2 subgroups according to their fibre diameter, $9-12 \mu \mathrm{m}$ on the one hand, and $18-20 \mu \mathrm{m}$ on the other hand, as already presented in Tables 1 and 2 .

\subsection{Materials out-of-plane permeability}

Studying the fluid transport behaviour in carbon non-wovens becomes of considerable interest as soon as their infiltration is considered, for producing composite materials for instance. Fibre architecture and surface chemistry, permeability and tortuosity, liquid viscosity and temperature are quantities known to influence the infiltration process of non-woven materials [39]. Therefore, any difference in fluid transport properties is important information to be known before selecting one or the other fibrous material for making composites. The corresponding values of out-of-plane permeability have been given in Table 2, and are presented in Fig. 4 as a function of either porosity or fibre diameter.

Indeed, as previously observed elsewhere [29,40,41], these two quantities are the main ones that affect fluid flow through fibrous porous media. The various structures of the present materials, however, did not allow observing any universal trend when all samples were considered together. Nevertheless, and as expected, porosity-dependent permeability could be observed within individual families and subgroups of materials, see Fig. 4(a). Thus, in most needle-punched soft felts, the permeability increased with the overall porosity. But clear deviations from this logical behaviour were also seen in the groups of Rayon-derived soft felts and thick-fibre PANderived soft felts, lower porosity sometimes leading to higher permeability. This might be explained by different manufacturing-induced morphological properties of the materials, especially in terms of number of fibre webs and of needle-punching density. 
(a)

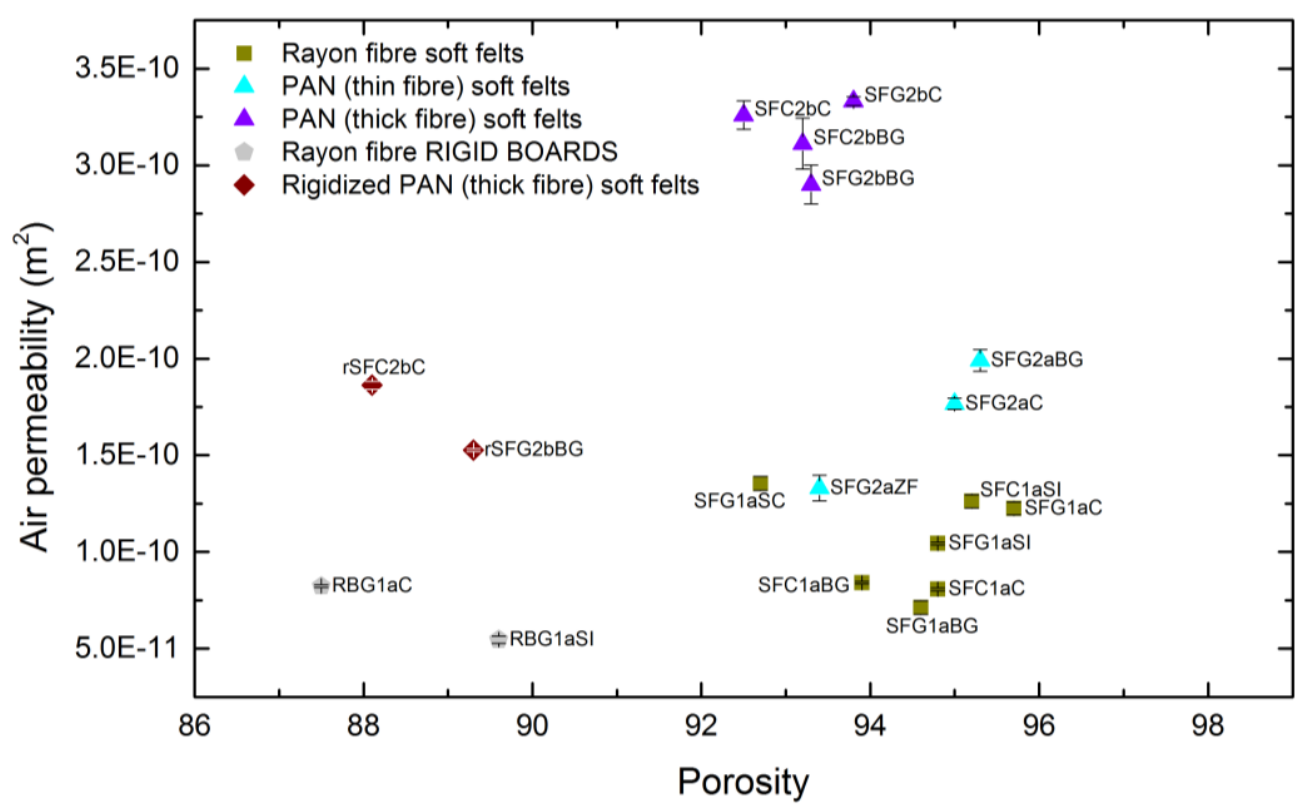

(b)

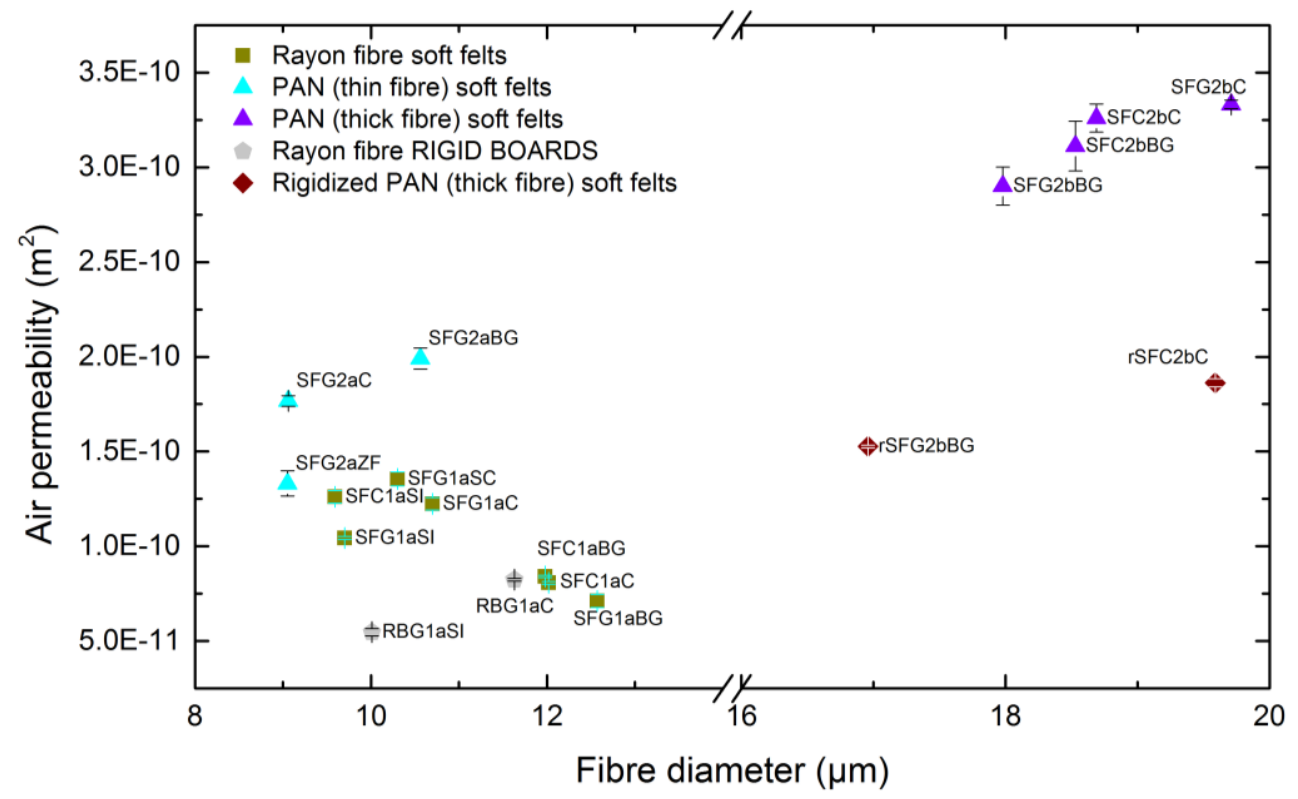

Fig. 4. Out-of-plane permeability of all materials, as a function of: (a) overall porosity, and (b) average fibre diameter. Each symbol represents a given carbon non-woven group, and the same symbols with different colours represent materials from different sub-groups of the same group. 
Due to their higher carbon fibre volume fraction, and to binder between fibres blocking and narrowing the pores, Rayon-derived rigid boards had lower permeability than most needlepunched soft felts, rigidized or not, as also observed elsewhere [13,39]. The same kind of conclusion applies to thick-fibre PAN-derived felts, the rigidized ones being clearly less permeable than the soft ones, which were free of chemical bonding. Furthermore, no increase of permeability with porosity was observed in the groups of Rayon-derived rigid boards and thickfibre PAN-derived rigidized soft felts. As previously observed in Fig. S5 of the Supplementary Information, these materials indeed differ in terms of distribution of fibre interconnections (compare $\mathrm{RBG1aC}$ with RBG1aSI), or in terms of felt formation and rigidizing technique (compare rSFG2bBG with rSFC2bC).

As for the effect of fibre diameter, shown in Fig. 4(b), opposite effects were observed: the permeability of rigidized and non-rigidized thick-fibre PAN-derived soft felts increased with fibre diameter, whereas that of Rayon-derived soft felts decreased. It can also be seen that Rayonderived materials presented lower permeability than their PAN-derived counterparts in similar ranges of porosity and fibre diameter. In addition to the aforementioned effect of the morphology differences induced by the production methods, the Rayon-derived fibres indeed present highly inhomogeneous surfaces and non-circular cross-sections compared to the smooth and almost cylindrical PAN-derived fibres (see again Fig. S6). All other things being equal, this may cause a higher drag to the fluid flow in Rayon-derived materials, thus lowering their permeability.

Comparing Fig. 4(a) and 4(b) also evidenced that the airflow permeability of materials made of thicker fibres is slightly higher than those made of thinner ones, whereas their porosity is generally lower than that of non-wovens made of thin fibres. Indeed, and as reported by other authors 
$[11,15]$, decreasing the fibre diameter increases their contact area and reduces the average pore size, all other things being constant.

Plotting the ratio of permeability $k$ to squared fibre radius $r^{2}$ thus appears to be a better option. Because it is dimensionless, $k / r^{2}$ is called reduced permeability, and it is the quantity most frequently used for characterising fluid flow through fibrous materials [16]. The corresponding graph is given in Fig. 5, and the data indeed appeared less scattered than in Fig. 4. However, given that several families of materials are considered together, still one single trend could not be observed, but a general trend appeared, according to which $k / r^{2}$ increases with $\varepsilon$. Attempts were made below to model such behaviour and to find a universal relationship embracing all measured values.

\section{Discussion}

\subsection{Theoretical background of permeability}

Generally speaking, three basic standard structures: granular, tubular and fibrous, have been considered from which permeability-porosity relationships have been suggested. The corresponding equations have been, and still are, improved by considering different approaches and refinements, among them the variational bounds theory, based on statistical correlation functions between pore structure and permeability [42], the percolation theory, which considers the porous medium as a random medium without spatial correlation [43], or the fractal theory, describing the porous structure with statistical geometrical self-similarities $[44,45]$. The roughness and the complexity of the pore surface, quantified by the fractal dimension of the porous medium under consideration [46], indeed has a strong impact on the permeability.

The well-known Kozeny-Carman equation [47] can take various forms such as: 


$$
k=\frac{\varepsilon\left(V_{p} / S_{p}\right)^{2}}{2 \eta_{v}}=\frac{\varepsilon r_{h}^{2}}{8 \eta_{v}}=\frac{\varepsilon r_{h}^{2}}{4 K_{c}}=\frac{\varepsilon^{3}}{K_{c} S_{b}^{2}}=\frac{\varepsilon^{3}}{K_{c} S_{S}^{2}(1-\varepsilon)^{2}}
$$

where $k$ and $\varepsilon$ are again the permeability and the porosity, respectively. $V_{p}$ and $S_{p}$ are the volume and the surface of the pore space, respectively. $\eta_{v}$ is the tortuosity factor for viscous flow, i.e., a ratio of the average fluid path through the porous material to material thickness, in viscous flow conditions. $r_{h}=2 V_{p} / S_{p}$ is the hydraulic radius. $K_{c}=2 \eta_{v}$ is called Kozeny constant $\left(K_{c}=2\right.$ for straight cylindrical tubes whereas $K_{c} \approx 5$ for many porous materials $) . S_{b}=\varepsilon /\left(V_{p} / S_{p}\right)$ is the internal accessible surface area per unit volume of bulk material, and $S_{S}$ is the same but expressed by unit volume of solid material so that $S_{s}=S_{b} /(1-\varepsilon)$. In the context of fractal mathematics, the KozenyCarman equation may also be written in the following, more general form [46]:

$$
k=C \frac{\varepsilon^{\alpha+2}}{(1-\varepsilon)^{n}}
$$

where $C$ is a coefficient including the geometrical parameters of the porous medium, $\alpha$ is called Archie's constant, and $n$ is a real number corresponding to the fractal dimension of the medium. It should be mentioned here that $n=1+\alpha[48]$, and hence the fractal dimension has the same physical meaning as Archie's exponent. By definition, Archie's exponent is directly related to the formation factor (see below) that allows estimating the tortuosity factor, for example. For geologists, it plays the same role as the cementation index [49] and, for mathematicians, the same as the fractal dimension.

Usually, the parameters $C, \alpha$ and $n$ are determined by fitting Eq. (5) to experimental data corresponding to different materials or materials categories (see for instance [50]). However, calculations show that estimating the permeability with such equations is much more difficult for a fibrous structure than for any porous medium. Indeed, a deviation between experimental and calculation results from Eq. (4) is observed for porosities higher than 0.2 [51]. This problem is 
related to the geometrical details of the porous medium such as shape, spatial distribution, orientation and volume fraction of fibres, having a significant impact on the tortuosity. Although tortuosity may have various definitions, depending on the considered phenomenon, this quantity is a way of describing the complexity of the structure of a porous material, i.e., how sinuous and interconnected it is [52]. Therefore, different fibrous geometries are still investigated today in order to get a deeper understanding and a better prediction of the structure-property relationships in materials models [51,53].

Nevertheless, some uncertainties and controversy have been reported. For example, Costa claimed that the value of Archie's exponent can vary from 1 to 4 in the case of the unified Kozeny-Carman equation applied to porous media, including fibrous ones [54]. Bayles et al. stipulated that the value of Archie's exponent can consistently be greater than unity, and that it is itself a function of both porosity and particle size distribution in the medium [48]. Tomadakis and Sotirchos suggested a generalised Archie's law in order to estimate, explicitly, the bulk diffusion tortuosity $\eta_{b}$ as a function of the porosity of the medium on the one hand, $\varepsilon$, and of the percolation threshold of the system on the other hand, $\varepsilon_{p}$, i.e., the critical porosity below which the fluid flow vanishes. The bulk tortuosity differs from the viscous one by the fact that the former is derived from Brownian diffusion random-walk simulation results in a porous medium, and not from viscous flow throughout the same medium. In the case of randomly overlapping fibres, the bulk diffusion tortuosity reads [55]:

$$
\eta_{b}=\left(\frac{1-\varepsilon_{p}}{\varepsilon-\varepsilon_{p}}\right)^{\alpha}
$$

and this kind of structure also imposes that [56]:

$$
r_{h}=-\frac{r}{\ln \varepsilon}
$$

where $r$ is again the fibre radius. 
Whereas the Kozeny-Carman model is based on a purely geometrical hydraulic radius, Johnson et al. developed a more accurate model based on a dynamical radius, $\Lambda$, which is an intrinsic measure of the dynamically interconnected pore size [57]. Therefore, it is a dynamical length scale directly related to transport, unlike the aforementioned hydraulic radius. In this context, Eq. (4) becomes:

$$
k=\frac{\Lambda^{2}}{8 F} \quad \text { where } \Lambda=-r_{h}\left(\frac{d \ln F}{d \ln \varepsilon}\right)^{-1} \quad \text { and } \quad F=\eta_{b} / \varepsilon
$$

$F$ is called formation factor and corresponds to the ratio of the bulk diffusivity of a chemical species in a fluid to the bulk diffusivity of this chemical species in a porous medium saturated with the same fluid. It thus represents a relative resistance to transport through a fluid with respect to a porous medium saturated with the same fluid.

Combining Eqs. (6), (7) and (8), one finally gets the permeability-porosity relationship for porous structures made of randomly overlapping fibres of mean circular radius $r$ [16]:

$$
\frac{k}{r^{2}}=\frac{\varepsilon}{8(\ln \varepsilon)^{2}}\left(\frac{\varepsilon-\varepsilon_{p}}{1-\varepsilon_{p}}\right)^{\alpha}\left(\frac{\varepsilon-\varepsilon_{p}}{(1+\alpha) \varepsilon-\varepsilon_{p}}\right)^{2}
$$

Eq. (9) behaves asymptotically like Eq. (5) when $\varepsilon_{p} \rightarrow 0, n=2$ and $\varepsilon \rightarrow 1$. Tomadakis and Robertson recommended the values of $\alpha$ and $\varepsilon_{p}$ reported in Table 3, depending on the structure of the fibrous medium and on the main direction of the flow through it [16]. For instance, for a fluid flow parallel to perfectly aligned fibres (i.e., the $1 \mathrm{D} / /$ problem in Table 3 ), $\alpha=\varepsilon_{p}=0$, thus the theoretical reduced permeability is the highest:

$$
\frac{k_{1 D / /}}{r^{2}}=\frac{\varepsilon}{8(\ln \varepsilon)^{2}}
$$

and the bulk tortuosity is obviously the lowest: $\eta_{b} 1 D / /=1$. In the other cases, the tortuosity is always higher than 1 , and for instance the tortuosity factor for viscous flow reads: 


$$
\eta_{v}=\frac{k_{1 D} / /}{k}>1
$$

Table 3. Archie's law parameters for the bulk diffusion tortuosity expressed by Eq. (6) [16].

\begin{tabular}{|c|c|c|c|c|}
\hline & Structure & Flow & $\varepsilon_{p}$ & $\alpha$ \\
\hline $1 \mathrm{D}$ & & $\begin{array}{l}/ / \text { to fibres } \\
\perp \text { to fibres }\end{array}$ & $\begin{array}{l}0 \\
0.33\end{array}$ & $\begin{array}{l}0 \\
0.707\end{array}$ \\
\hline $2 \mathrm{D}$ & & $\begin{array}{l}/ / \text { to fibres layers } \\
\perp \text { to fibres layers }\end{array}$ & $\begin{array}{l}0.11 \\
0.11\end{array}$ & $\begin{array}{l}0.521 \\
0.785\end{array}$ \\
\hline $3 \mathrm{D}$ & & All directions & 0.037 & 0.661 \\
\hline
\end{tabular}

The Tomadakis - Robertson equation (9) is probably the most efficient one for fitting the permeability-porosity relationship of a number of fibrous media, as observed in many former studies [5,41,58-60]. For this reason, it will receive a special attention in the next subsection. But former models should also be considered. For instance, the empirical model of Davies [61], represented by Eq. (12), is supposed to fit the OP permeability data of highly porous fibrous materials whose porosity $\varepsilon$ is higher than $70 \%$ :

$$
\frac{k}{r^{2}}=\left\{16(1-\varepsilon)^{\frac{3}{2}}\left[1+56(1-\varepsilon)^{3}\right]\right\}^{-1}
$$

Tamayol and Bahrami [62] investigated the OP permeability of several fibrous structures involving square, staggered, and hexagonal arrangements of unidirectional aligned fibres, along with simple 2D felts and simple cubic matrices. They proposed the following analytical equation: 


$$
\frac{k}{r^{2}}=0.032 \sqrt{\varepsilon}\left[\left(\frac{\pi}{4(1-\varepsilon)}\right)^{2}-\left(\frac{\pi}{2(1-\varepsilon)}\right)+1\right]
$$

Van Doormaal and Pharoah [63] used a Lattice Boltzmann approach for simulating a fluid flow through idealised porous layers and a Monte Carlo method for generating their geometry. The resultant calculated permeability of random fibrous porous materials was fitted by the following equation:

$$
\frac{k}{r^{2}}=0.28 \frac{\varepsilon^{4.3}}{1-\varepsilon}
$$

Other fractal and mechanistic models have also been proposed by the group of Fan and Ding [64-68] and are not reported in detail here, although they have been tested and confronted to our experimental results, as explained below.

\subsection{Application of the models to the experimental results}

The values of reduced permeability measured by Gostick et al. on commercial fibrous carbon gas diffusion layers for fuel cells [5] were introduced in Fig. 5, in addition to the present results for the 18 carbon non-wovens. As can be seen, no model was able to correctly fit a broad variety of materials, whose different structures explain why it happened that samples having higher porosities than others could have lower reduced permeabilities, and vice-versa. The model of Davies (Eq. (12)) underestimated the reduced permeability of all materials except the group of Rayon-derived soft felts (see Fig. 5(a)). The underestimation can be explained by the drag theory [41], according to which the model does not take into account the lower drag performed from the fibres oriented along the fluid flow. The overestimation seen in the case of Rayon-derived needlepunched soft carbon felts is explained by the preferential orientation of fibres perpendicular to the OP direction and the higher drag due to non-circular fibre cross-sections and rough fibre surfaces. 
(a)

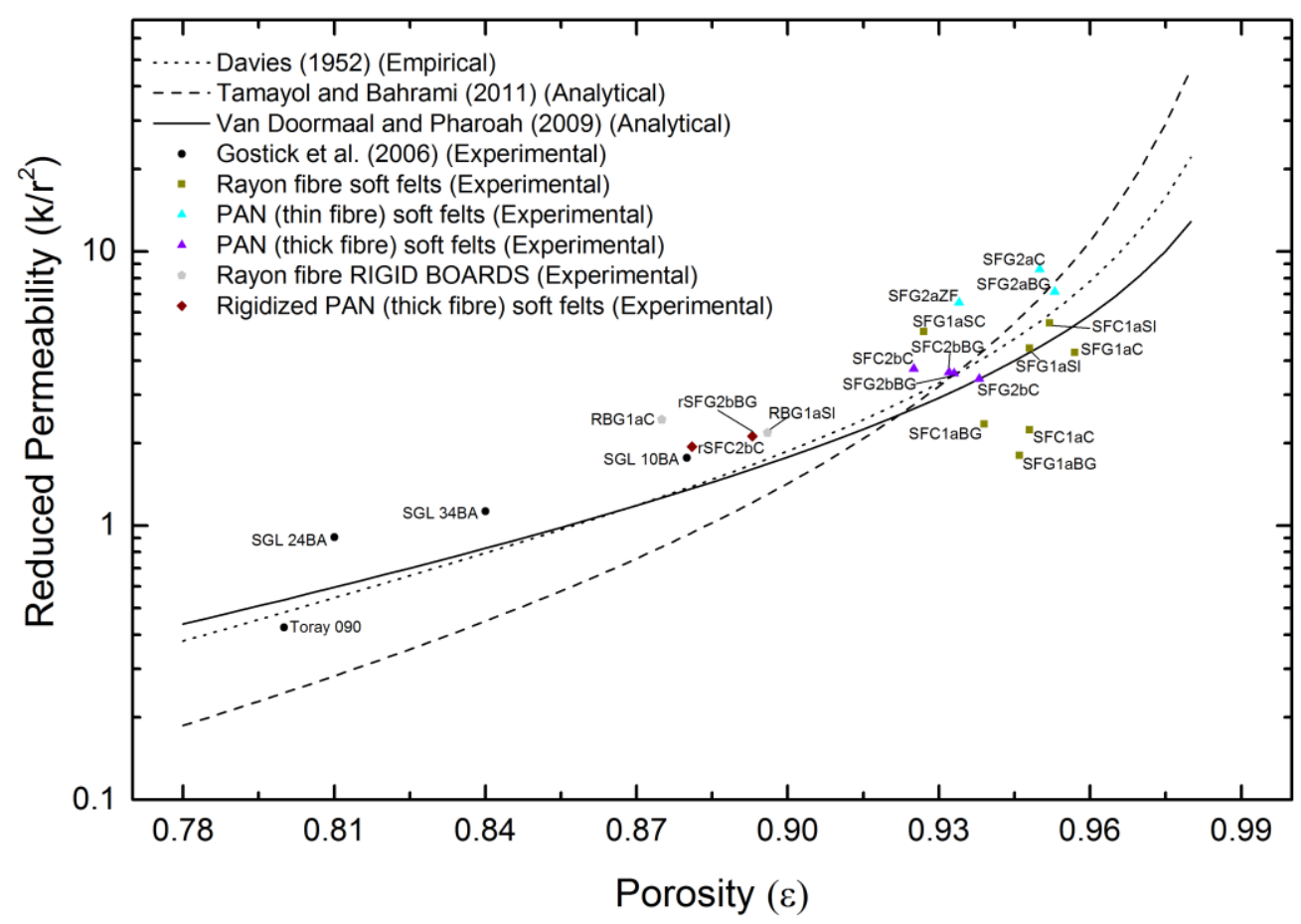

(b)

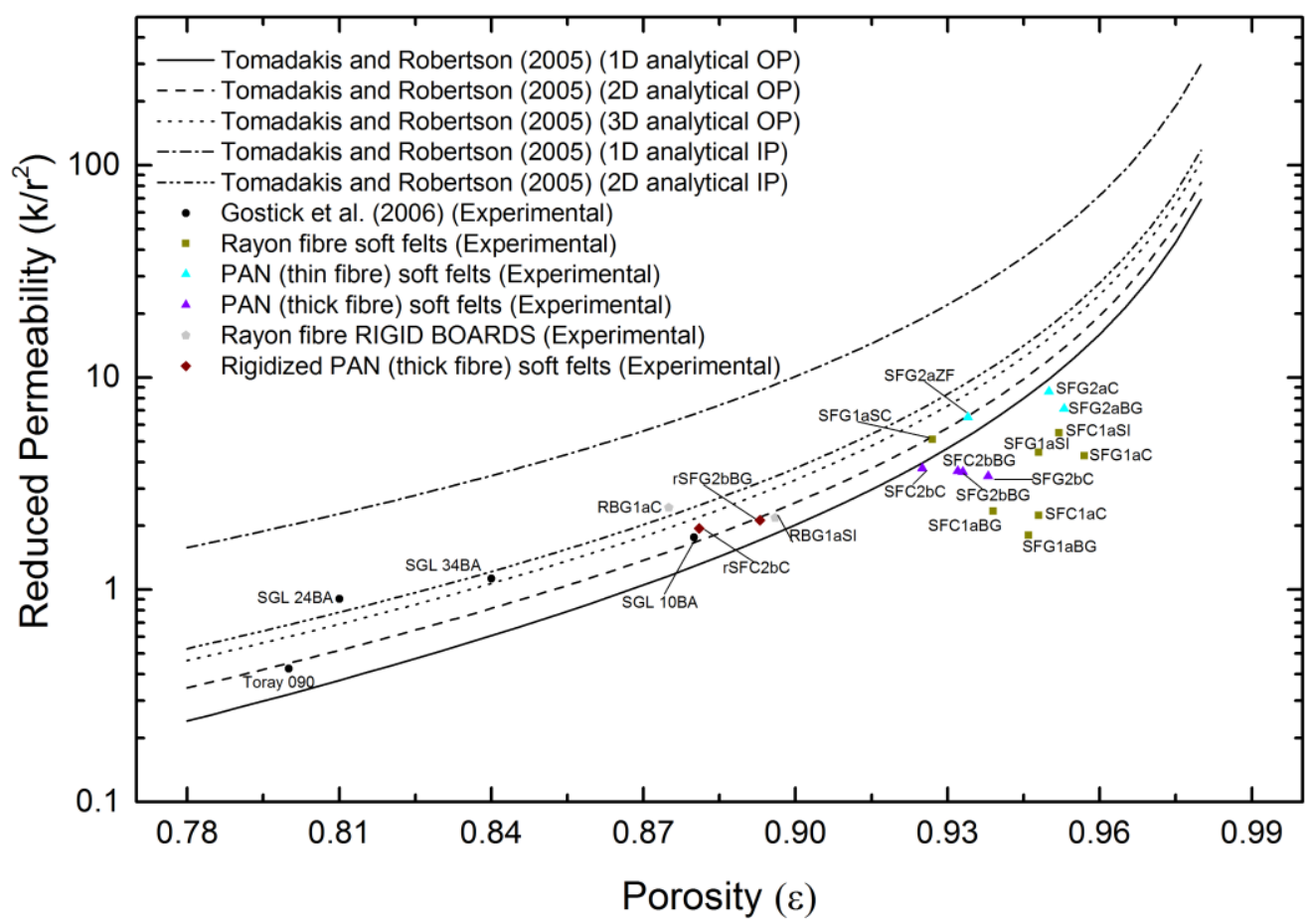

Fig. 5. Out-of-plane reduced permeability of all present materials as well as a few other ones from the literature, as a function of overall porosity. The symbols have the same meaning as in Fig. 4. The curves correspond to the application of models of: (a) Davies (Eq. (12)), Tamayol - Bahrami (Eq. (13)), and Van Doormaal - Pharoah (Eq. (14)); and (b) Tomadakis - Robertson (Eq. (9)). 
The same kind of conclusions also applies to the Tamayol - Bahrami model (Eq. (13)), for which the discrepancy between experimental and calculated values was found to be even higher (see again Fig. 5(a)). These deviations are based on the differences between the model's assumption of perfectly layered structures, with fibres randomly oriented along the IP direction and layered orthogonally to the fluid flow, and the real non-woven materials structure, with random deflections in the fibre orientation. As previously shown in $[41,45,62]$, and although most of the present materials have porosities outside the range formerly simulated in the original paper, the model of Van Doormaal - Pharoah (Eq. (14)) was worth testing as well because it refers to various porous transport layers including carbon fibre nonwoven materials having a typical fibre diameter ranging from 7 to $12 \mu \mathrm{m}$. This model led to the curve having the lowest slope, thus passing closer to most groups of carbon non-wovens, and hence producing the best compromise for the whole set of needle-punched soft felts (at a porosity $\varepsilon$ higher than $92 \%$ ). This can be explained by the very realistic approach of the simulation of porous layers made by Van Doormaal and Pharoah [63]. Moreover, these authors indicated that structures made of orthogonal neighbouring layers of randomly oriented fibres are a good approximation of random fibre structures, as proved by the statistical similarity of their OP permeability estimations.

Testing the models of Fan and Ding [64-68] produced exactly the same kind of curves as those shown in Fig. 5. In other words, no improvement of the fits was obtained, as seen in Fig. S7 of the Electronic Supplementary Information. The models indeed preserved the shape of the evolution of the reduced permeability as a function of porosity in a manner similar to the other analytical models presented in the manuscript. However, we have observed that due to some morphological differences, samples with higher porosities than others may have reduced permeabilities, and viceversa. Therefore, as we have seen above, the suggested models remain very dependent on fitting 
parameters, thus allow estimating correctly the permeability of the materials only at low or high porosity, and not both at once.

Finally, and unlike most other research works dealing with one family of fibrous mats having different porosities, fitting Eq. (9) with the parameters of Table 3 was again impossible for all materials together, as it clearly overestimated the reduced permeability in most cases, see Fig. 5(b). The agreement between experimental and predicted values was only fair in the range of low to medium porosities investigated here, and especially in the case of thick-fibre PAN-derived rigidized soft felts and Rayon-derived rigid boards, considering these materials as intermediates between 2D $\perp$ and 3D random structures (see again Table 3). The reduced permeability of 3 materials: rSFG2bBG, SFG1aSC and SFG2aZF, was even exactly the one predicted by the 3D Tomadakis - Robertson model. The feature shared by these materials is their high needlepunching density observed by SEM, although the latter could not be quantified. These results suggest that some needle-punched and/or chemically bonded samples can be well represented by Eq. (9), as previously observed by Soltani et al. [69]. The rest of the investigated materials reduced permeability values was definitely lower than what was predicted by any form of Eq. (9), including the one corresponding to the $1 \mathrm{D} \perp$ structure, which leads to the lowest possible values.

Nevertheless, it should be stressed that finding Tomadakis' model as the most suitable one is logical since the corresponding equation contains the largest number of free parameters such as $\varepsilon$ and $F$, themselves containing implicitly Archie's exponent or fractal dimension (see again Section 4.1). The other models are all derived from the unified Kozeny-Carman equation with $\alpha$ fixed by fitting and with only one free parameter, $\varepsilon$. Besides, Tomadakis' model also considers $\varepsilon_{p}$, which is not the case for the other models and this is the reason why it better fits the experimental values collected from the literature. However, in the case of the most porous materials, the calculated 
reduced permeability is still overestimated, as seen in Fig. 5(b). When $\varepsilon \rightarrow 1$, Tomadakis' model converges towards its asymptotic value $C /(1-\varepsilon)^{n}$, where $C$ is the same coefficient as in Eq. (5) and where $n=2$. The discrepancy observed in Fig. 5(b) at high porosity thus means that the values of $C$ and $n$ are not the right ones for our materials when $\varepsilon \rightarrow 1$, and hence highly porous fibrous carbons are not correctly represented by the standard 1D, 2D and 3D "flow structures" shown in Table 3 .

Moreover, the multi-layered consolidated structure of the present fibrous carbon materials provides a much higher resistance to air flow. Such superior resistance should correspond to a significantly increased tortuosity factor, and indeed, the only way of shifting downwards the curves shown in Fig. 5(b) is using values of $\alpha$ that are much higher than those given in Table 3. The Archie's exponent was then calculated as follows. For randomly overlapping fibre structures the combination of Eqs. (4) and (7) leads to:

$$
\frac{k}{r^{2}}=\frac{\varepsilon}{8 \eta_{v}(\ln \varepsilon)^{2}}
$$

and since the Kozeny constant $K_{c}$ is equal to $2 \eta_{v}$, it reads:

$$
K_{c}=\frac{\varepsilon r^{2}}{4 k(\ln \varepsilon)^{2}}
$$

The corresponding data can be readily calculated, given that $\varepsilon, k$ and $r$ have been measured and were given in Table 2. Now, the expression of the tortuosity factor can be found by identification of Eqs. (9) and (15):

$$
\eta_{v}=\left[\left(\frac{\varepsilon-\varepsilon_{p}}{1-\varepsilon_{p}}\right)^{\alpha}\left(\frac{\varepsilon-\varepsilon_{p}}{(1+\alpha) \varepsilon-\varepsilon_{p}}\right)^{-2}\right]^{-1}=\eta_{b}\left(1+\frac{\alpha \varepsilon}{\varepsilon-\varepsilon_{p}}\right)^{2}
$$

Given the very high porosity values of the present materials, already listed in Table 2 and ranging from $\sim 88$ to $\sim 96 \%$, the percolation threshold $\varepsilon_{p}$ can be considered as negligible in Eq. (17). Such 
approximation was already successfully used for describing the physical properties of highly porous graphite materials [70]. Eq. (17) thus simplifies into:

$$
\eta_{v}=\frac{(1+\alpha)^{2}}{\varepsilon^{\alpha}}
$$

so the Archie's exponent $\alpha$ is the solution of the final equation:

$$
\frac{(1+\alpha)^{2}}{\varepsilon^{\alpha}}=\frac{K_{c}}{2}=\frac{\varepsilon r^{2}}{8 k(\ln \varepsilon)^{2}}
$$

The mathematical analysis revealed that the obtained solution is always unique within $[0 ;+\infty[$. Resolving Eq. (19) for each of our fibrous carbon materials led to the results given in Fig. 6, where $\alpha$ has been plotted as a function of $\eta_{v}$.

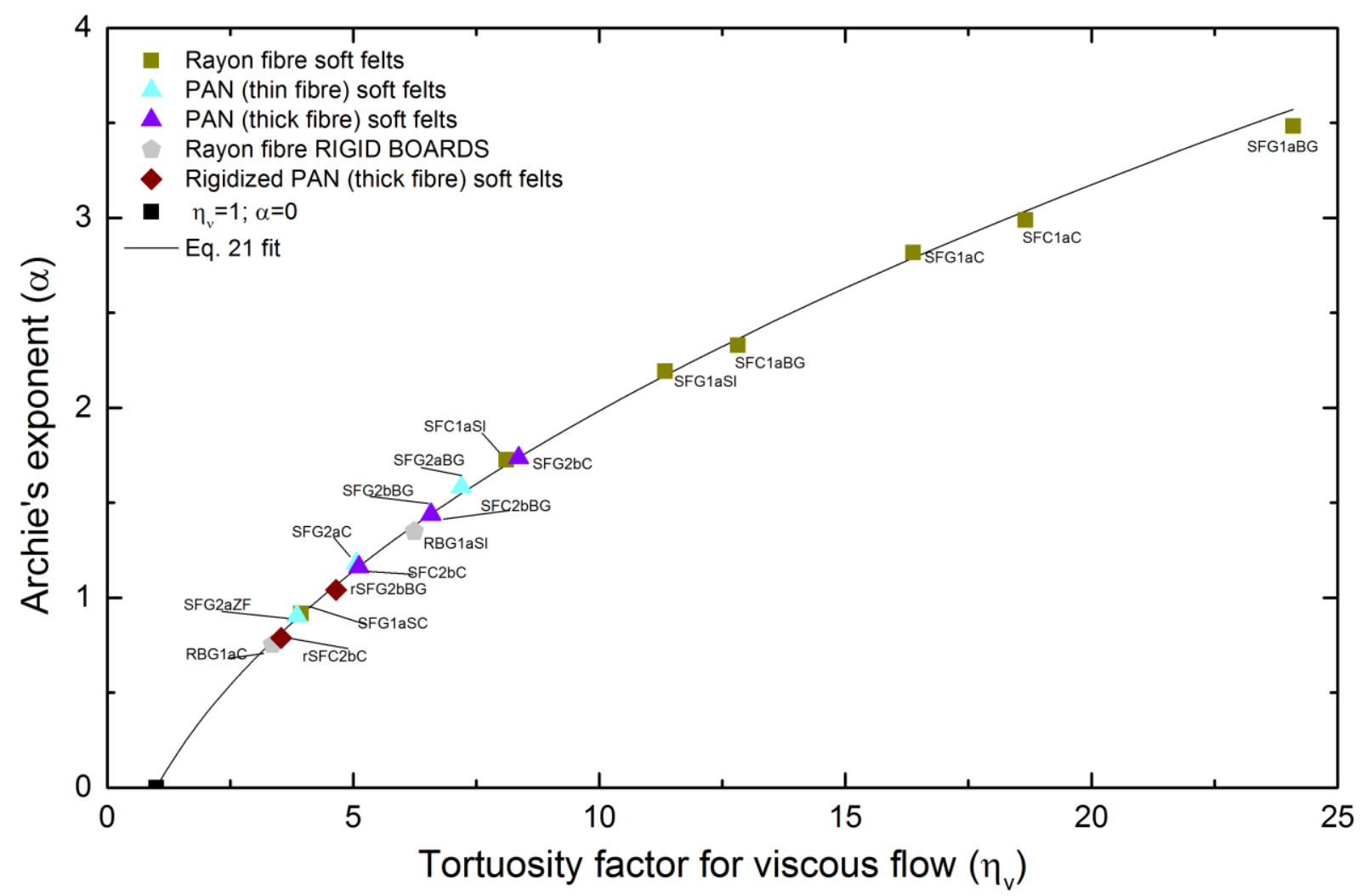

Fig. 6. Archie's coefficient, $\alpha$, vs. viscous tortuosity factor, $\eta_{v}$, for all fibrous carbon materials investigated here, calculated from Eq. (19), i.e., assuming that the percolation threshold is zero. 
The solid line is a fit of Eq. (21) to the data points, plus one added at $\left(\eta_{v}=1 ; \alpha=0\right)$. The symbols have the same meaning as in Fig. 4, plus one added for $\left(\eta_{v}=1 ; \alpha=0\right)$.

It is usually accepted that the Archie's exponent, or cementation index, is close to 1.5 [49]. It is indeed the case most frequently encountered with many materials [71,72], and it is even exactly equal to $3 / 2$ for a dilute assembly of spheres [73]. In the present case, it can be seen that, whereas half of tested materials presented Archie's exponent in the range $1-2$, values as high as almost 3.5 were also found. The same kind of comment can be done for the Kozeny constant: whereas its value is close to 5 for most common materials, values in the range $7-14$ were reported for more complex granular materials having fractal geometry [48]. The present materials are definitely more complex than granular ones, thereby leading to lower permeabilities, since $K_{c}$ ranged from roughly 7 to 50 , i.e., varied by almost one order of magnitude. This finding agrees with former results for which the values of $K_{c}$ were even found to span over two orders of magnitude [16].

Fig. 6 shows that despite so many different families of materials are gathered in one single plot, all data points fall on the same master curve. This finding strongly suggests that $\alpha$ is not a free parameter but may be considered as an intrinsic property of the fibrous medium. To the best of the authors' knowledge, this is the first time that the master curve shown in Fig. 6 is obtained. It could be fitted with a very good accuracy in the range of investigated values (determination factor $R^{2}=$ $0.99895)$ by a second-order polynomial such that:

$$
\alpha=0.08435+0.2264 \eta_{v}-0.00362 \eta_{v}{ }^{2}
$$

To further improve the quality of the prediction, one additional point was added at $\alpha=0$, for which $\eta_{v}=\eta_{b}=1$. With such additional data, for which no real material exists, the polynomial fit was slightly less perfect, whereas the following relationship was truly excellent instead (determination coefficient $R^{2}=0.99958$ ): 


$$
\alpha=-1.0967+1.0725 \eta_{v}{ }^{0.45805}
$$

The same numerical treatment was also applied to the experimental data points presented by Jackson and James [50] for fibrous materials having a range of porosities even broader than that of our own samples, i.e., such that $0.346 \leq \varepsilon \leq 0.995$. The result is given in Fig. 7, which clearly demonstrates that the aforementioned master curve can be extended to many other different fibrous materials. Thus, in contrast to other studies, the fibres average radius $r$, the porosity $\varepsilon$ and the permeability $k$ have been measured, from which $K_{c}$ and hence $\eta_{v}$ could be obtained from Eq. (19). Then, the solution of Eq. (18) led to the values of $\alpha$. In other words, $\alpha$ is now experimentally determined and no more numerically fixed, as it was the case in former works. The main limitation of this analysis is the fact that if data are too noisy, then the scattering of $\alpha$ values may become very important. However, a very good alignment of data can be seen in Fig. 7, despite the extreme diversity of the samples, suggesting that materials should be classified according to their increasing value of $\alpha$. 


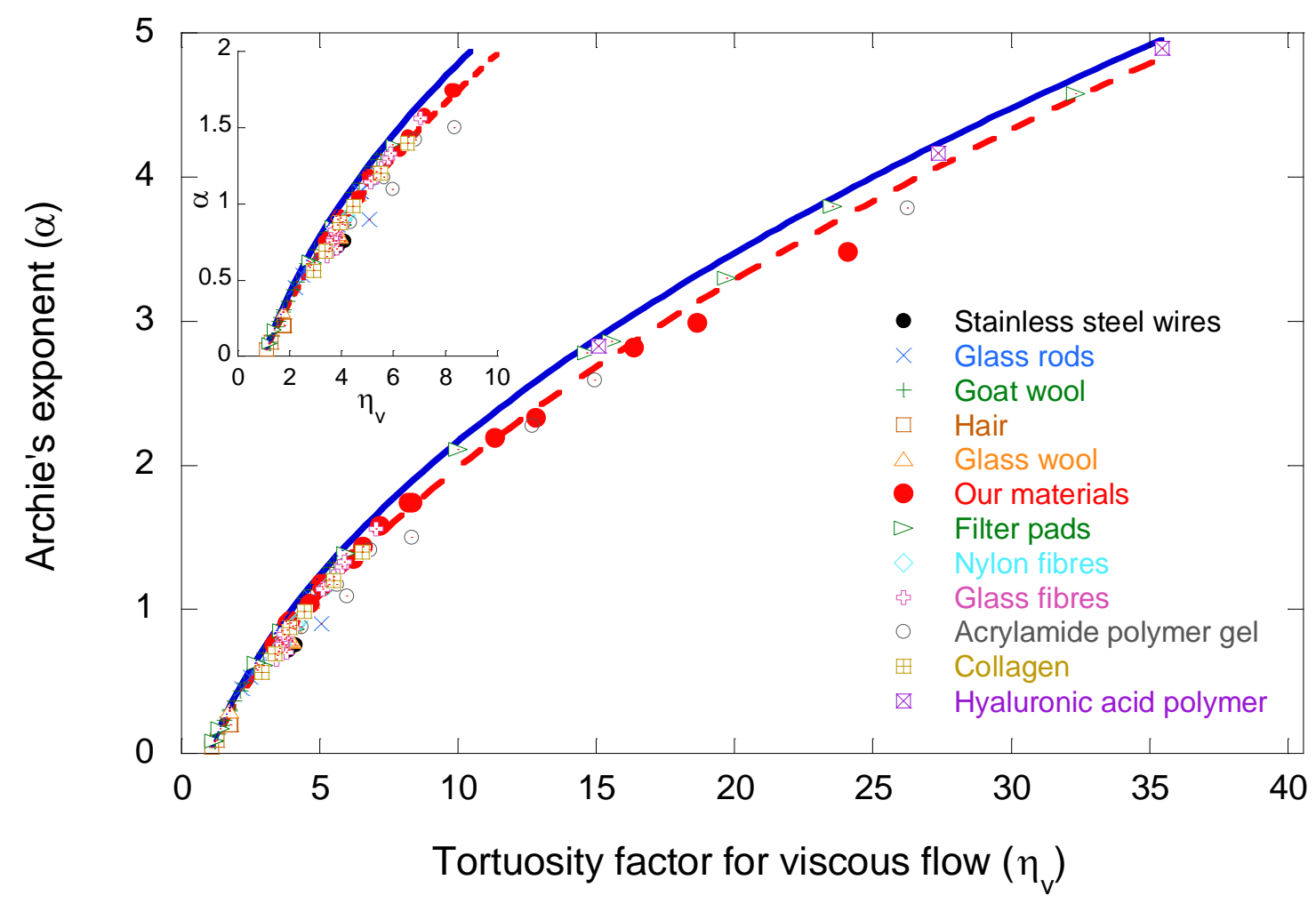

Fig. 7. Same as Fig. 6, but with the addition of 106 other fibrous materials referenced on the plot, having porosity within the range 34.6 up to $99.5 \%$ and whose data were taken from [50]. The red dashed line is the fit of Eq. (22) to the whole set of data, whereas the solid blue line corresponds to the boundary expressed by Eq. (23). The inset is a zoom on the lowest values of $\alpha$ and $\eta_{v}$.

A fit of the form of Eq. (21) was tried again on the whole set of data, thus encompassing all samples including our own ones, with a determination coefficient of 0.99669 :

$$
\alpha=-0.7405+0.73836 \eta_{v}^{0.56635}
$$

Broadening this way the ranges of both porosities and materials for applying the same kind of fit obviously led to a slight decrease of accuracy in the more restricted domain of fibrous carbons, but demonstrates that the clear correlation between Archie's coefficient and tortuosity still holds. Eq. (21) should thus be preferred as long as usual fibrous carbons are concerned. 
Of course, Eqs. (20) to (22) are expected to be valid only for tortuosity factors $\eta_{v} \geq 1$. It can be seen that Eqs. (21) and (22) are close to the simple expression:

$$
\alpha=-1+\eta_{v} 0.5
$$

or, in other words, they suggest that:

$$
\eta_{v}=(1+\alpha)^{2}
$$

which is indeed the lower boundary of the viscous tortuosity when $\varepsilon \rightarrow 1$ (see again Eq. (18)). In Fig. 7, the data corresponding to such limit have been reported, and it can be clearly seen that all experimental data are either on or below this limiting curve.

The combination of Eqs. (17) and (24) suggests that $\eta_{b}$ should be always close to 1 in our series of materials. Calculating $\eta_{b}$ from Archie's law, which is the simplest form of Eq. (6) when the percolation threshold $\varepsilon_{p}$ is assumed to be zero, and which thus reads:

$$
\eta_{b}=\varepsilon^{-\alpha}
$$

indeed led to values within the range $1.06-1.21$ (see also Fig. 8 below).

Thus, Archie's exponent is defined only by the tortuosity factor $\eta_{b}$, and hence allows the calculation of the formation factor $F=\eta_{b} / \varepsilon$. The corresponding results are shown in Fig. 8 . 
(a)

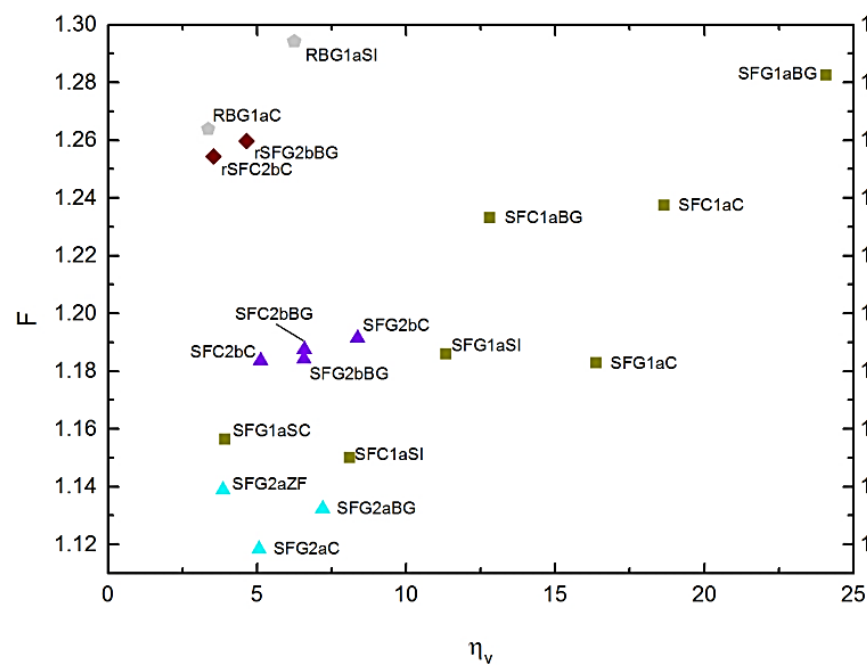

(b)

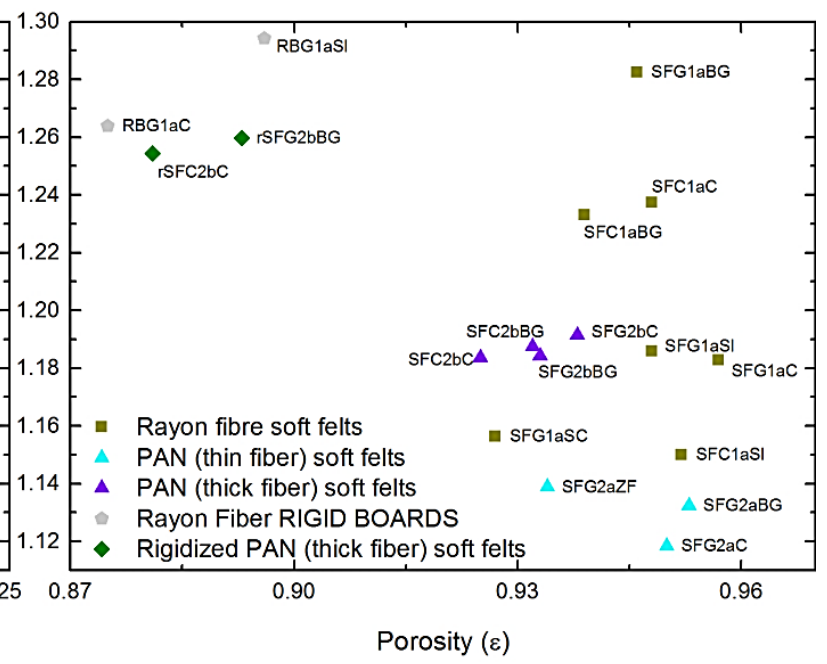

Fig. 8. Formation factor $F$ calculated from the data of Fig. 6 and from the known porosity of the materials (see again Eq. (8)), and plotted as a function of: (a) tortuosity factor for viscous flow, $\eta_{v}$; and (b) porosity, $\varepsilon$. The symbols have the same meaning as in Fig. 4.

Fig. 8 illustrates the broad variety of fibrous carbons considered here, since no trend could be observed. For instance, some materials were both highly porous and highly tortuous at the same time, such as all Rayon-derived soft felts except SFG1aSC, whereas others were both less porous and less tortuous, especially $\mathrm{RBG} 1 \mathrm{aC}$ or $\mathrm{rSFC} 2 \mathrm{bC}$. Such radically opposite behaviours are explained by the structure, whose randomness can lead to high tortuosity despite the high porosity on the one hand, or whose high needle-punching density and/or numerous fibres clumps, clusters and bundles formations can lead to many straight canals throughout the material and hence to a lower tortuosity despite a lower porosity on the other hand. Of course, intermediate situations exist for which lower tortuosities correspond to higher porosities (e.g., SFG2aC, SFG2aBG and to a lower extent SFG1aSC and SFG2aZF). But for most materials, the tortuosity globally increased with the porosity, as seen in Fig. 9. 


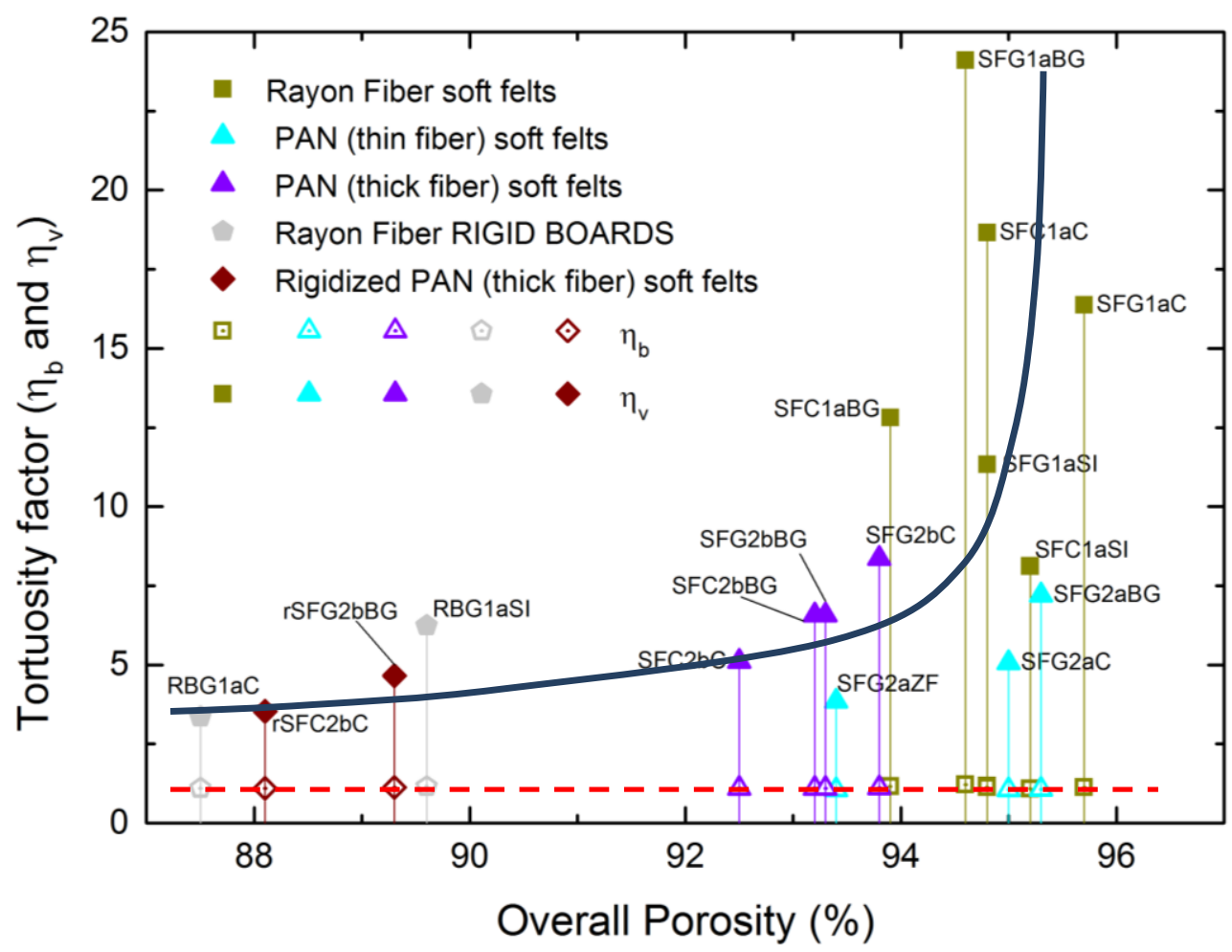

Fig. 9. Tortuosity factors of all samples, as a function of their overall porosity. The symbols have the same meaning as in Fig. 4. Solid and empty symbols stand for $\eta_{v}$ and $\eta_{b}$, respectively. The corresponding full and dashed lines, respectively, are just guides for the eye.

The general trend that can be seen in Fig. 9 for $\eta_{v}$ is totally counter-intuitive as long as materials of similar structure are considered. Moreover, the corresponding Kozeny's constants $\left(K_{c}\right.$ $=2 \eta_{v}$ ) are far higher than those predicted by Tomadakis and Roberson [16] using the fixed parameters of Table 3. But in the case of carbon felts, and as recalled above, the structure is controlled by the need of maintaining a mechanical integrity of these materials: those of higher porosity have a more deformable character, so that a higher mechanical consolidation (hence a higher entanglement of fibres) is required, leading to a much more complex and intricate structure. The same applies to thick materials, whatever their porosity, made by consolidation of successive layers of fibrous mats. 
Given that the results presented in Figs. 6 - 9 were obtained from the assumption that the percolation threshold is negligible, a sensitivity analysis was also carried out. First, a few non-zero values of $\varepsilon_{p}$ were tested, and how the values of Archie's exponent changed with the viscous tortuosity factor was calculated. The results are shown in Fig. S8(a) of the Electronic Supplementary Information. It can be seen that using $\varepsilon_{p}=0.037$, i.e., the value expected for random 3D materials (see again Table 3) very poorly affected the Archie's exponent, especially in the range of low to moderate tortuosity factors. Values as high as $\varepsilon_{p}=0.11$, i.e., corresponding to purely 2D materials (which is unrealistic in our case: see again Figs. S1 to S5) led to higher changes (up to one unit) but again at extreme values of tortuosities only. The impact of a non-zero percolation threshold should thus be considered as negligible, and especially for materials having porosities close to 1 .

Finally, the same kind of sensitivity analysis was carried out as for the impact of the porosity, assuming again that $\varepsilon_{p}=0$. It can be seen in Fig. S8(b) that $\alpha$ was strongly affected by the value of $\varepsilon$. Nevertheless, the couples of values $\left(\alpha, \eta_{v}\right)$ strictly remained on the same master curve as the one presented in Fig. 6, thus supporting its robustness and suggesting that $\alpha$ should be considered as an intrinsic property of the porous medium and not as constants, as formerly recommended by Tomadakis and Robertson.

\section{Conclusion}

In this work, 18 commercial fibrous carbons ranging from soft felts to rigid boards through rigidized felts were thoroughly investigated in terms of general structure, total porosity, average fibre diameter, and through-thickness air permeability. These materials were derived from either PAN or Rayon fibres having different diameters, were graphitised or not, and consolidated with various techniques usually employed in the industry, i.e., either mechanically (through needle- 
punching, assisted or not with an additional chemical bonding) or chemically with a resin subsequently carbonised. As a result, a full set of materials with rather different porous structures and hence different permeabilities, irrespective to their porosity, was considered.

Attempts carried out for sorting the materials by groups and sub-groups of closely related samples revealed consistent behaviours within limited ranges of structures or porosities, but were unable to account for all observations. The huge scattering of the results from one family of materials to another was explained by the versatility of structures, so that no model could be fitted to the whole set of samples. And especially, all models dramatically overestimated the reduced permeability in the high-porosity range. This finding also applied to the very popular TomadakisSotirchos equation, despite its higher number of parameters, and which is widely used to describe the permeability of carbon felts used as electrodes in redox flow batteries or as gas diffusion layers in fuel cells, for instance.

The Archie's coefficient and the viscous tortuosity (through the Kozeny constant) were then calculated from measured values of porosity, fibre diameter and permeability. A clear correlation between these two parameters was evidenced, so that all data were perfectly aligned on one single master curve. The analysis was then successfully extended to many other fibrous materials, and all the data from the literature were also found to follow the same master curve. The Archie's coefficient thus appears to be an intrinsic property, purely defined by the material geometry, as it does not depend on the 1D, 2D or 3D-type of flow. In contrast, the parameters in Tomadakis' model were fixed and therefore not relevant to a broad range of fibrous structures. A fitting equation was proposed, encompassing all fibrous materials in very broad ranges of porosities and structures. Based on this new equation, all fibrous materials can be classified according to their Archie's coefficient, thus leading to an outstanding predicting character. 


\section{Acknowledgements}

This work was partly supported by a grant overseen by the French National Research Agency (Pc2TES ANR-16-CE06-0012-01). The authors are indebted to all people having kindly supplied free samples for our studies: Sean McFeely from Ceramaterials (UK), Zhao (Johnson) Xi from Beijing Great Wall Co. Ltd (China), Alain Pichon from SGL CARBON S.A.S. (France), Francis Guérard and Elena Ogneva from Schunk Electrographite (France), and István Fáykiss and Kinga

Kincses from Zoltek (Hungary). The authors also gratefully acknowledge the financial support of the CPER 2007-2013 "Structuration du Pôle de Compétitivité Fibres Grand'Est" (Competitiveness Fibre Cluster, France), through local (Conseil Général des Vosges), regional (Région Lorraine), national (DRRT and FNADT) and European (FEDER, France) funds.

\section{Conflict of interest}

The authors declare no conflict of interest toward any individual or organisation. 


\section{References}

[1] A.E. Scheidegger (1958) The physics of flow through porous media, Soil Sci. 86.

[2] F.A.L. Dullien (1992) Porous media: fluid transport and pore structure, 2nd ed., Academic Press, San Diego, CA.

[3] Z. Chen, R.E. Ewing (2002) Fluid flow and transport in porous media: mathematical and numerical treatment, American Mathematical Society. doi:http://dx.doi.org/10.1090/conm/295.

[4] M. Sahimi (2011) Flow and transport in porous media and fractured rock: from classical methods to modern approaches, John Wiley \& Sons, Hoboken, NJ.

[5] J.T. Gostick, M.W. Fowler, M.D. Pritzker, M.A. Ioannidis, L.M. Behra (2006) In-plane and through-plane gas permeability of carbon fiber electrode backing layers, J. Power Sources. 162:228-238. doi:10.1016/j.jpowsour.2006.06.096.

[6] J. Ihonen, M. Mikkola, G. Lindbergh (2004) Flooding of gas diffusion backing in PEFCs physical and electrochemical characterization, J. Electrochem. Soc. 151:A1152-A1161. doi:10.1149/1.1763138.

[7] H. Dohle, R. Jung, N. Kimiaie, J. Mergel, M. Müller (2003) Interaction between the diffusion layer and the flow field of polymer electrolyte fuel cells-experiments and simulation studies, J. Power Sources. 124:371-384. doi:10.1016/S0378-7753(03)00800-0.

[8] J.H. Chun, K.T. Park, D.H. Jo, S.G. Kim, S.H. Kim (2011) Numerical modeling and experimental study of the influence of GDL properties on performance in a PEMFC, Int. J. Hydrog. Energy. 36:1837-1845. doi:10.1016/j.ijhydene.2010.01.036.

[9] M.V. Williams, E. Begg, L. Bonville, H.R. Kunz, J.M. Fenton (2004) Characterization of gas diffusion layers for PEMFC, J. Electrochem. Soc. 151:A1173-A1180. doi:10.1149/1.1764779. 
[10] B. Mueller, T. Zawodzinski, J. Bauman, F. Uribe, S. Gottesfeld, E.D. Castro, et al. (1998) Carbon cloth gas diffusion backings for high performance PEFC cathodes, ECS Proc. Vol. 1998-27:1-9. doi:10.1149/199827.0001PV.

[11] C.J. Hung, C.H. Liu, T.H. Ko, W.H. Chen, S.H. Cheng, W.S. Chen, et al. (2013) Effect of diffusion layers fabricated with different fiber diameters on the performance of low temperature proton exchange membrane fuel cells, J. Power Sources. 221:134-140. doi:10.1016/j.jpowsour.2012.08.030.

[12] M. Prasanna, H.Y. Ha, E.A. Cho, S.-A. Hong, I.-H. Oh (2004) Influence of cathode gas diffusion media on the performance of the PEMFCs, J. Power Sources. 131:147-154. doi:10.1016/j.jpowsour.2004.01.030.

[13] A. El-kharouf, T.J. Mason, D.J.L. Brett, B.G. Pollet (2012) Ex-situ characterisation of gas diffusion layers for proton exchange membrane fuel cells, J. Power Sources. 218:393-404. doi:10.1016/j.jpowsour.2012.06.099.

[14] P. Mangal, L.M. Pant, N. Carrigy, M. Dumontier, V. Zingan, S. Mitra, et al. (2015) Experimental study of mass transport in PEMFCs: through plane permeability and molecular diffusivity in GDLs, Electrochimica Acta. 167:160-171. doi:10.1016/j.electacta.2015.03.100.

[15] D.M. Fadzillah, M.I. Rosli, M.Z.M. Talib, S.K. Kamarudin, W.R.W. Daud (2017) Review on microstructure modelling of a gas diffusion layer for proton exchange membrane fuel cells, Renew. Sustain. Energy Rev. 77:1001-1009. doi:10.1016/j.rser.2016.11.235.

[16] M.M. Tomadakis, T.J. Robertson (2005) Viscous permeability of random fiber structures: comparison of electrical and diffusional estimates with experimental and analytical results, J. Compos. Mater. 39:163-188. doi:10.1177/0021998305046438. 
[17] J.H. Nam, M. Kaviany (2003) Effective diffusivity and water-saturation distribution in single- and two-layer PEMFC diffusion medium, Int. J. Heat Mass Transf. 46:4595-4611. doi:10.1016/S0017-9310(03)00305-3.

[18] T.X. Huong Le, M. Bechelany, M. Cretin (2017) Carbon felt based-electrodes for energy and environmental applications: A review, Carbon. 122:564-591. doi:10.1016/j.carbon.2017.06.078.

[19] T.J. Kang, K.H. Jung, J.K. Park, J.R. Youn, S.G. Lee (2002) Effect of punching density on the mechanical and thermal properties of needle-punched nonwoven carbon/phenolic composites, Polym. Compos. 10:521-530. doi:10.1177/096739110201000704.

[20] J. Lee, L. Yun, J. Park (2005) Anisotropic properties of needle punched carbon/carbon composites, 18th international conference on composite materials. 1-4.

[21] W. Shi, J.Y. Li, Q.F. You, T. Lu, Y. Tan (2015) Preparation and properties of rigid carbon felt thermal insulation, Mater. Sci. Forum. 833:48-51. doi:10.4028/www.scientific.net/MSF.833.48.

[22] H. Cheng, C. Hong, X. Zhang, H. Xue (2015) Lightweight carbon-bonded carbon fiber composites with quasi-layered and network structure, Mater. Des. 86:156-159. doi:10.1016/j.matdes.2015.07.091.

[23] C. Prabha Karan (2016) Nonwovens: process, structure, properties and applications, Woodhead Publishing India PVT. Limited.

[24] Y. Zhang, Z. Lu, Z. Yang, D. Zhang, J. Shi, Z. Yuan, Q. Liu (2017) Compression behaviors of carbon-bonded carbon fiber composites: Experimental and numerical investigations, Carbon. 116:398-408. doi:10.1016/j.carbon.2017.02.012.

[25] S. Russell (2006) Handbook of Nonwovens - 1st Edition. 
[26] H.-G. Geus (2016) 5 - Developments in manufacturing techniques for technical nonwovens, In: G. Kellie (Ed.), Adv. Tech. Nonwovens, Woodhead Publishing, pp 133-153. doi:10.1016/B978-0-08-100575-0.00005-X.

[27] M.S.A. Rahaman, A.F. Ismail, A. Mustafa (2007) A review of heat treatment on polyacrylonitrile fiber, Polym. Degrad. Stab. 92:1421-1432. doi:10.1016/j.polymdegradstab.2007.03.023.

[28] X. Huang (2009) Fabrication and properties of carbon fibers, Materials. 2:2369-2403. doi:10.3390/ma2042369.

[29] M.D.R. Kok, J.T. Gostick (2015) Transport properties of electrospun fibrous membranes with controlled anisotropy, J. Membr. Sci. 473:237-244. doi:10.1016/j.memsci.2014.09.017.

[30] N. Mao (2016) 6 - Methods for characterisation of nonwoven structure, property, and performance, In: G. Kellie (Ed.), Adv. Tech. Nonwovens, Woodhead Publishing, pp 155211. doi:10.1016/B978-0-08-100575-0.00006-1.

[31] M.S. Dresselhaus (1988) Graphite fibers and filaments, Berlin, Allemagne, France.

[32] S. Zhong, C. Padeste, M. Kazacos, M. Skyllas-Kazacos (1993) Comparison of the physical, chemical and electrochemical properties of rayon- and polyacrylonitrile-based graphite felt electrodes, J. Power Sources. 45:29-41. doi:10.1016/0378-7753(93)80006-B.

[33] S. Liu, M. Kok, Y. Kim, J.L. Barton, F.R. Brushett, J. Gostick (2017) Evaluation of electrospun fibrous mats targeted for use as flow battery electrodes, J. Electrochem. Soc. 164:A2038-A2048. doi:10.1149/2.1301709jes.

[34] F. Dullien (2012) Porous media fluid transport and pore structure, Elsevier, Burlington, MA.

[35] P. Soltani, M.S. Johari, M. Zarrebini (2015) Tomography-based determination of transverse permeability in fibrous porous media, J. Ind. Text. 44:738-756. doi:10.1177/1528083713512357. 
[36] F. Canbolat (2011) Nonwovens bonding technologies: an overview on mechanisms, properties, and application fields, Electron. J. Text. Technol. 5:54-60.

[37] F. Panerai, J.C. Ferguson, J. Lachaud, A. Martin, M.J. Gasch, N.N. Mansour (2017) Microtomography based analysis of thermal conductivity, diffusivity and oxidation behavior of rigid and flexible fibrous insulators, Int. J. Heat Mass Transf. 108:801-811. doi:10.1016/j.ijheatmasstransfer.2016.12.048.

[38] B.A. Newcomb (2016) Processing, structure, and properties of carbon fibers, Compos. Part Appl. Sci. Manuf. 91:262-282. doi:10.1016/j.compositesa.2016.10.018.

[39] S.-J. Park (2015) Carbon Fibers, Springer Netherlands.

[40] P. Soltani, M.S. Johari, M. Zarrebini (2014) Effect of 3D fiber orientation on permeability of realistic fibrous porous networks, Powder Technol. 254:44-56. doi:10.1016/j.powtec.2014.01.001.

[41] P. Soltani, M. Zarrebini, R. Laghaei, A. Hassanpour (2017) Prediction of permeability of realistic and virtual layered nonwovens using combined application of X-ray $\mu \mathrm{CT}$ and computer simulation, Chem. Eng. Res. Des. 124:299-312. doi:10.1016/j.cherd.2017.06.035.

[42] S. Prager (1961) Viscous flow through porous media, Phys. Fluids. 4:1477-1482. doi:10.1063/1.1706246.

[43] A. Hunt (2009) R. Ewing, Percolation theory for flow in porous media, 2nd ed. 2009 edition, Springer, Berlin.

[44] M. Sahimi (1990) Y.C. Yortsos, Applications of fractal geometry to porous media: a review.

[45] J. Cai, L. Luo, R. Ye, X. Zeng, X. Hu (2015) Recent advances on fractal modeling of permeability for fibrous porous media, Fractals. 23:1-9. doi:10.1142/S0218348X1540006X. 
[46] N. Henderson, J.C. Brêttas, W.F. Sacco (2010) A three-parameter Kozeny-Carman generalized equation for fractal porous media, Chem. Eng. Sci. 65:4432-4442. doi:10.1016/j.ces.2010.04.006.

[47] P.C. Carman (1937) Fluid flow through granular beds, Trans Inst Chem Eng. 15:150-166.

[48] G.A. Bayles, G.E. Klinzing, S.-H. Chiang (1989) Fractal mathematics applied to flow in porous systems, Part. Syst. Charact. 6:168-175. doi:10.1002/ppsc.19890060128.

[49] P.N. Sen, C. Scala, M.H. Cohen (1981) A self-similar model for sedimentary rocks with application to the dielectric constant of fused glass beads, Geophysics. 46:781-795. doi:10.1190/1.1441215.

[50] G.W. Jackson, D.F. James (1986) The permeability of fibrous porous media, Can. J. Chem. Eng. 64:364-374. doi:10.1002/cjce.5450640302.

[51] F. Xiao, X. Yin (2016) Geometry models of porous media based on Voronoi tessellations and their porosity-permeability relations, Comput. Math. Appl. 72:328-348. doi:10.1016/j.camwa.2015.09.009.

[52] M. Letellier, V. Fierro, A. Pizzi, A. Celzard (2014) Tortuosity studies of cellular vitreous carbon foams, Carbon. 80:193-202. doi:10.1016/j.carbon.2014.08.056.

[53] S. Bargmann, B. Klusemann, J. Markmann, J.E. Schnabel, K. Schneider, C. Soyarslan, J. Wilmers (2018) Generation of 3D representative volume elements for heterogeneous materials: A review, Prog. Mater. Sci. 96:322-384. doi:10.1016/j.pmatsci.2018.02.003.

[54] A. Costa (2006) Permeability-porosity relationship: a reexamination of the Kozeny-Carman equation based on a fractal pore-space geometry assumption, Geophys. Res. Lett. 33 . doi:10.1029/2005GL025134.

[55] M.M. Tomadakis, S.V. Sotirchos (1993) Ordinary and transition regime diffusion in random fiber structures, AIChE J. 39:397-412. doi:10.1002/aic.690390304. 
[56] M.M. Tomadakis, S.V. Sotirchos (1991) Effective Kundsen diffusivities in structures of randomly overlapping fibers, AIChE J. 37:74-86. doi:10.1002/aic.690370107.

[57] D.L. Johnson, J. Koplik, R. Dashen (1987) Theory of dynamic permeability and tortuosity in fluid-saturated porous media, J. Fluid Mech. 176:379-402. doi:10.1017/S0022112087000727.

[58] N. Zamel, X. Li (2013) Effective transport properties for polymer electrolyte membrane fuel cells - with a focus on the gas diffusion layer, Prog. Energy Combust. Sci. 39:111-146. doi:10.1016/j.pecs.2012.07.002.

[59] X. Ke, J.M. Prahl, J.I.D. Alexander, R.F. Savinell (2018) Redox flow batteries with serpentine flow fields: distributions of electrolyte flow reactant penetration into the porous carbon electrodes and effects on performance, J. Power Sources. 384:295-302. doi:10.1016/j.jpowsour.2018.03.001.

[60] S. Simaafrookhteh, M. Shakeri, M. Baniassadi, A.A. Sahraei (2018) Microstructure reconstruction and characterization of the porous GDLs for PEMFC based on fibers orientation distribution, Fuel Cells. 18:160-172. doi:10.1002/fuce.201700239.

[61] C.N. Davies (1953) The separation of airborne dust and particles, Proc. Inst. Mech. Eng. Part B Manag. Eng. Manuf. 1:185-213. doi:10.1177/095440545300100113.

[62] A. Tamayol, M. Bahrami (2011) Transverse permeability of fibrous porous media, Phys. Rev. E. 83:046314. doi:10.1103/PhysRevE.83.046314:1-9.

[63] M.A.V. Doormaal, J.G. Pharoah (2009) Determination of permeability in fibrous porous media using the lattice Boltzmann method with application to PEM fuel cells, Int. J. Numer. Methods Fluids. 59:75-89. doi:10.1002/fld.1811.

[64] D. Shou, J. Fan, F. Ding (2010) A difference-fractal model for the permeability of fibrous porous media, Phys. Lett. A. 374:1201-1204. doi:10.1016/j.physleta.2010.01.002. 
[65] D. Shou, J. Fan, F. Ding (2011) Hydraulic permeability of fibrous porous media, Int. J. Heat Mass Transf. 54:4009-4018. doi:10.1016/j.ijheatmasstransfer.2011.04.022.

[66] B. Xiao, J. Fan, F. Ding (2012) Prediction of Relative Permeability of Unsaturated Porous Media Based on Fractal Theory and Monte Carlo Simulation, Energy Fuels. 26:6971-6978. doi:10.1021/ef3013322.

[67] D. Shou, Y. Tang, L. Ye, J. Fan, F. Ding (2013) Effective permeability of gas diffusion layer in proton exchange membrane fuel cells, Int. J. Hydrog. Energy. 38:10519-10526. doi:10.1016/j.ijhydene.2013.06.043.

[68] B. Xiao, J. Fan, F. Ding (2014) A fractal analytical model for the permeabilities of fibrous gas diffusion layer in proton exchange membrane fuel cells, Electrochimica Acta. 134:222231. doi:10.1016/j.electacta.2014.04.138.

[69] P. Soltani, M.S. Johari, M. Zarrebini (2013) Tomography-based determination of transverse permeability in fibrous porous media, J. Ind. Text. 44:738-756. doi:10.1177/1528083713512357.

[70] A. Celzard, J.F. Marêché, G. Furdin (2003) Describing the properties of compressed expanded graphite through power laws, J. Phys. Condens. Matter. 15:7213-7226. doi:10.1088/0953-8984/15/43/006.

[71] P. Wong, J. Koplik, J.P. Tomanic (1984) Conductivity and permeability of rocks, Phys. Rev. B. 30:6606-6614. doi:10.1103/PhysRevB.30.6606.

[72] A. Celzard, J.F. Marêché (2002) Fluid flow in highly porous anisotropic graphites, J. Phys. Condens. Matter. 14:1119-1129. doi:10.1088/0953-8984/14/6/301.

[73] Groupe Poreux PC (1987) Transport in heterogeneous porous media, Phys. Scr. T19B:524530. doi:10.1088/0031-8949/1987/T19B/033. 